\title{
1 Evaluating and optimizing the use of diagnostics during epidemics: 2 Application to the 2017 plague outbreak in Madagascar
}

3 Quirine ten Bosch ${ }^{1,2^{*}}$, Voahangy Andrianaivoarimanana ${ }^{3^{*}}$, Beza Ramasindrazana ${ }^{3^{*}}$, Guillain Mikaty ${ }^{4}$, 4 Rado JL Rakotonanahary ${ }^{3}$, Birgit Nikolay ${ }^{1}$, Soloandry Rahajandraibe ${ }^{3}$, Maxence Feher ${ }^{4}$, Quentin

5 Grassin $^{4}$, Juliette Paireau ${ }^{1}$, Soanandrasana Rahelinirina ${ }^{3}$, Rindra Randremanana ${ }^{5}$, Feno

Rakotoarimanana ${ }^{5}$, Marie Melocco ${ }^{5}$, Voahangy Rasolofo ${ }^{6}$, Javier Pizarro-Cerda ${ }^{7,8,9}$, Anne-Sophie Le Guern $^{7,8,9}$, Eric Bertherat ${ }^{10}$, Maherisoa Ratsitorahina ${ }^{6,11}$, André Spiegel ${ }^{6}$, Laurence Bari ${ }^{5+}$, Minoarisoa Rajerison $^{3+}$, Simon Cauchemez ${ }^{1+}$

*: Equal contribution

${ }^{+}$: Joint senior contribution

${ }^{1}$ Mathematical Modelling of Infectious Diseases Unit, Institut Pasteur, UMR2000, CNRS, Paris, France

${ }^{2}$ Quantitative Veterinary Epidemiology, Department of Animal Sciences, Wageningen University and Research, Wageningen, the Netherlands

${ }^{3}$ Plague Unit, Institut Pasteur de Madagascar, Antananarivo, Madagascar

${ }^{4}$ Environment and Infectious risks research unit, Laboratory for Urgent Response to Biological Threats (ERI-CIBU), Institut Pasteur, Paris, France

${ }^{5}$ Epidemiology and Clinical Research Unit, Institut Pasteur de Madagascar, Antananarivo, Madagascar

${ }^{6}$ Direction, Institut Pasteur de Madagascar, Antananarivo, Madagascar

${ }^{7}$ Yersinia Research Unit, Institut Pasteur, Paris, France

${ }^{8}$ National Reference Laboratory for Plague and other yersinioses, Institut Pasteur, Paris, France

${ }^{9}$ World Health Organization Collaborating Center, Institut Pasteur, Paris, France

${ }^{10}$ World Health Organization, Health Emergency Programme, Department of Infectious Hazard Management, Geneva, Switzerland

${ }^{11}$ Directorate of Health and Epidemiological Surveillance, Ministry of Public Health, Antananarivo, Madagascar

\section{Email: quirine.tenbosch@wur.nl}

Keywords: Plague, Yersinia Pestis, Epidemiology, Outbreak Response, Diagnostics, Latent Class, Modeling

This PDF file includes: 


\section{Abstract}

During outbreaks, the lack of diagnostic "gold standard" can mask the true burden of infection in the population and hamper the allocation of resources required for control. Here, we present an analytical framework to evaluate and optimize the use of diagnostics when multiple yet imperfect diagnostic tests are available. We apply it to laboratory results of 2,136 samples, analyzed with three diagnostic tests (based on up to seven diagnostic outcomes), collected during the 2017 pneumonic (PP) and bubonic plague (BP) outbreak in Madagascar, which was unprecedented both in the number of notified cases, clinical presentation, and spatial distribution. The extent of this outbreaks has however remained unclear due to non-optimal assays. Using latent class methods, we estimate that $7 \%-15 \%$ of notified cases were Yersinia pestis-infected. Overreporting was highest during the peak of the outbreak and lowest in the rural settings endemic to Yersinia pestis. Molecular biology methods offered the best compromise between sensitivity and specificity. The specificity of the rapid diagnostic test was relatively low (PP: 82\%, BP: 85\%), particularly for use in contexts with large quantities of misclassified cases. Comparison with data from a subsequent seasonal Yersinia pestis outbreak in 2018 reveal better test performance (BP: specificity 99\%, sensitivity: 91\%), indicating that factors related to the response to a large, explosive outbreak may well have affected test performance. We used our framework to optimize the case classification and derive consolidated epidemic trends. Our approach may help reduce uncertainties in other outbreaks where diagnostics are imperfect.

\section{Introduction}

The availability of accurate diagnostics is essential for an effective response to infectious disease outbreaks. In the relatively common situation where no gold standard diagnostic is available (i.e. absence of a diagnostic test with perfect sensitivity and specificity), interpretation of diagnostic results becomes challenging $(1,2)$. This may hamper case identification and management, jeopardize the evaluation of the burden, scope, timing, and spatial expansion of the outbreak, and ultimately impede control. Here, taking a large plague outbreak in Madagascar as a case study, we present an integrative analytical framework to assess the performance of diagnostics and reconstruct spatio-temporal epidemic patterns in situations where multiple yet imperfect diagnostics are available.

Plague is a highly fatal disease caused by a gram-negative bacillus Yersinia pestis (3). Rodents constitute its natural reservoir and the bacillus can be transmitted to humans by fleas. When bitten by an infected flea, a person typically develops bubonic plague (BP), which is characterized by fever and painful lymphadenitis in the area of the fleabite (3). Septicemic spread can occasionally lead to pneumonic plague (PP) that typically consists of sudden fever, cough, and symptoms of lower respiratory tract infections. Interhuman transmission of PP is possible through droplet spread (4). Plague case fatality ratio (CFR) has been estimated between $10-40 \%$ (5-7). Diagnosis, particularly of PP, is challenging due to i) non-specific early symptoms $(8,9)$, ii) the difficulty to collect high-quality sputum samples, especially from severely ill and young patients $(10)$, iii) the scarcity of PP cases hampering evaluation of diagnostics; most assays have been evaluated on BP samples (11).

Between August and November 2017, Madagascar experienced a large number $(2,414)$ of notifications of clinically suspected plague cases that were predominantly in two major urban areas $(79 \%)$ with unusually high proportions of PP (78\%) (Fig 1A,B) (12). Important discrepancies between tests (the proportion of positive PP results ranged from $1 \%$ to $18 \%$ depending on the test; Fig $1 C, D$ ) means that 
the true extent of the PP outbreak remains unclear. Besides, without a good understanding of the performances of the diagnostics available, it is difficult to optimize diagnostic and case classification algorithms for future outbreaks. Here, we analyze data describing this large plague epidemic to obtain a comprehensive view of the burden of infection among notified cases. We evaluate the performance of test diagnostics and propose updated case-classification algorithms to better allocated sparse resources during future outbreaks. Using the combined test results and diagnostic performance estimates, we reconstruct epidemiological trends over space and time.
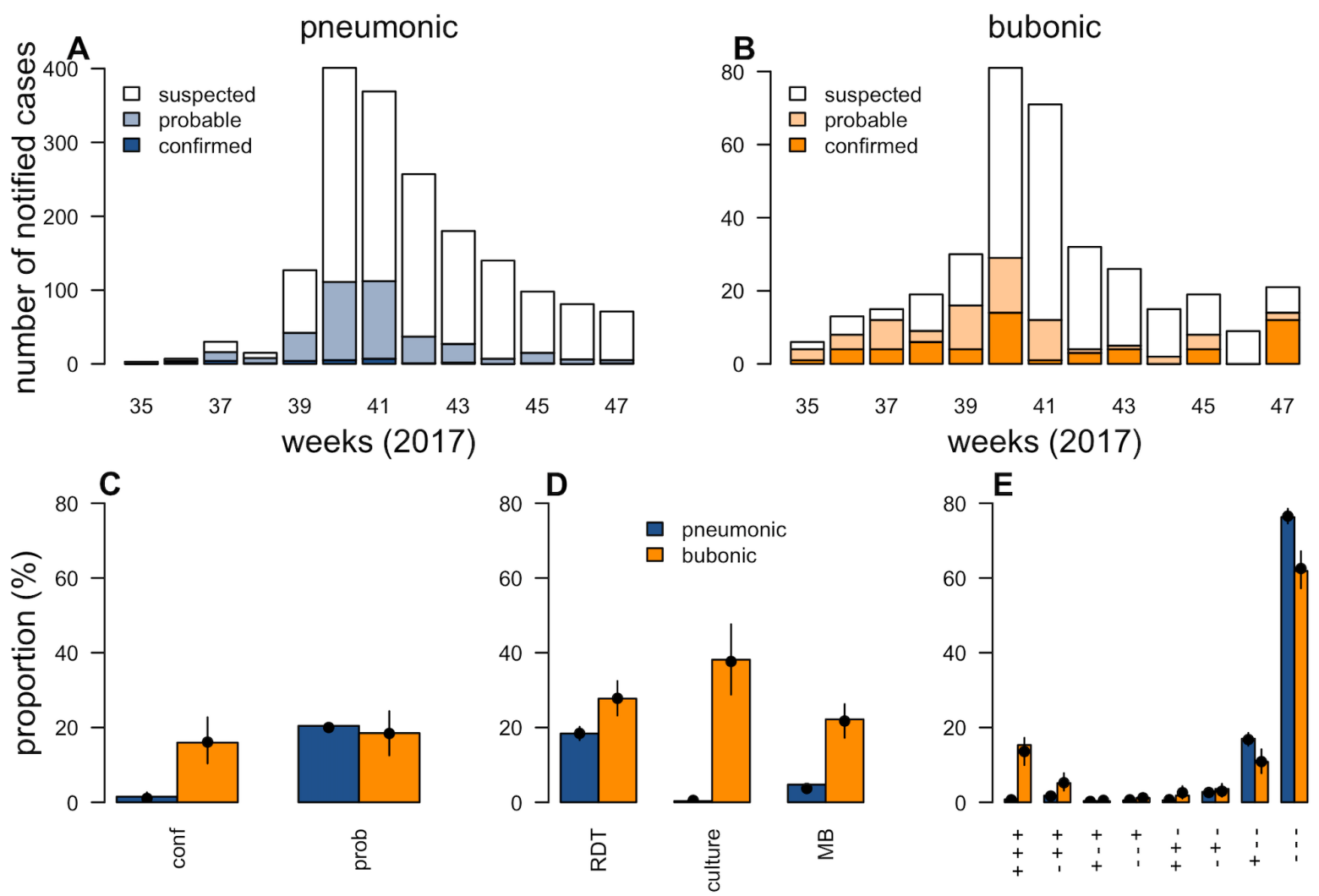

Figure 1. Diagnostics and case classification during the plague outbreak in Madagascar in 2017. (A,B): Weekly number of notified cases for pneumonic plague (A) and bubonic plague (B) by case classification. (C,D,E): Proportion of notified cases classified as confirmed (conf) or probable (prob) (C), with a positive test result for RDT, culture, or MB (NB. only cases on whom the respective test was performed are considered in the denominator. No restrictions were put on the use of $M B$ and RDT. Culture was only performed if RDT was positive, apart from PP samples from non-endemic regions. On those samples, culture was performed irrespective of RDT result) (D) and with a certain combination of diagnostic outcomes (E), presenting outcomes that were performed on all samples (RDT, qPCR on pla and caf1 genes). Model fits to these proportions are provided with black dots and lines indicating model predictions and $95 \%$ credible intervals, respectively.

\section{Results}

Of 2,414 notifications, we consider those with sputum or bubo aspirates and known clinical form (pulmonary plague (PP): 1,779, bubonic plague (BP): 357) (12). Of PP sputum samples, $22 \%$ have at least one positive culture $(N=4)$, rapid diagnostic test (RDT) ( $N=327)$, or molecular biology (MB) $(N=84)$ (Fig 1D) and are classified, based on their diagnostic outcomes (Fig 2), as either confirmed (2\%) or probable (20\%) (Fig 1C), vs. $34 \%$ of BP (37 culture, $99 \mathrm{RDT}, 79 \mathrm{MB}$ ) (Fig 1D) with $16 \%$ confirmed and $18 \%$ probable (Fig 1C) (12). 


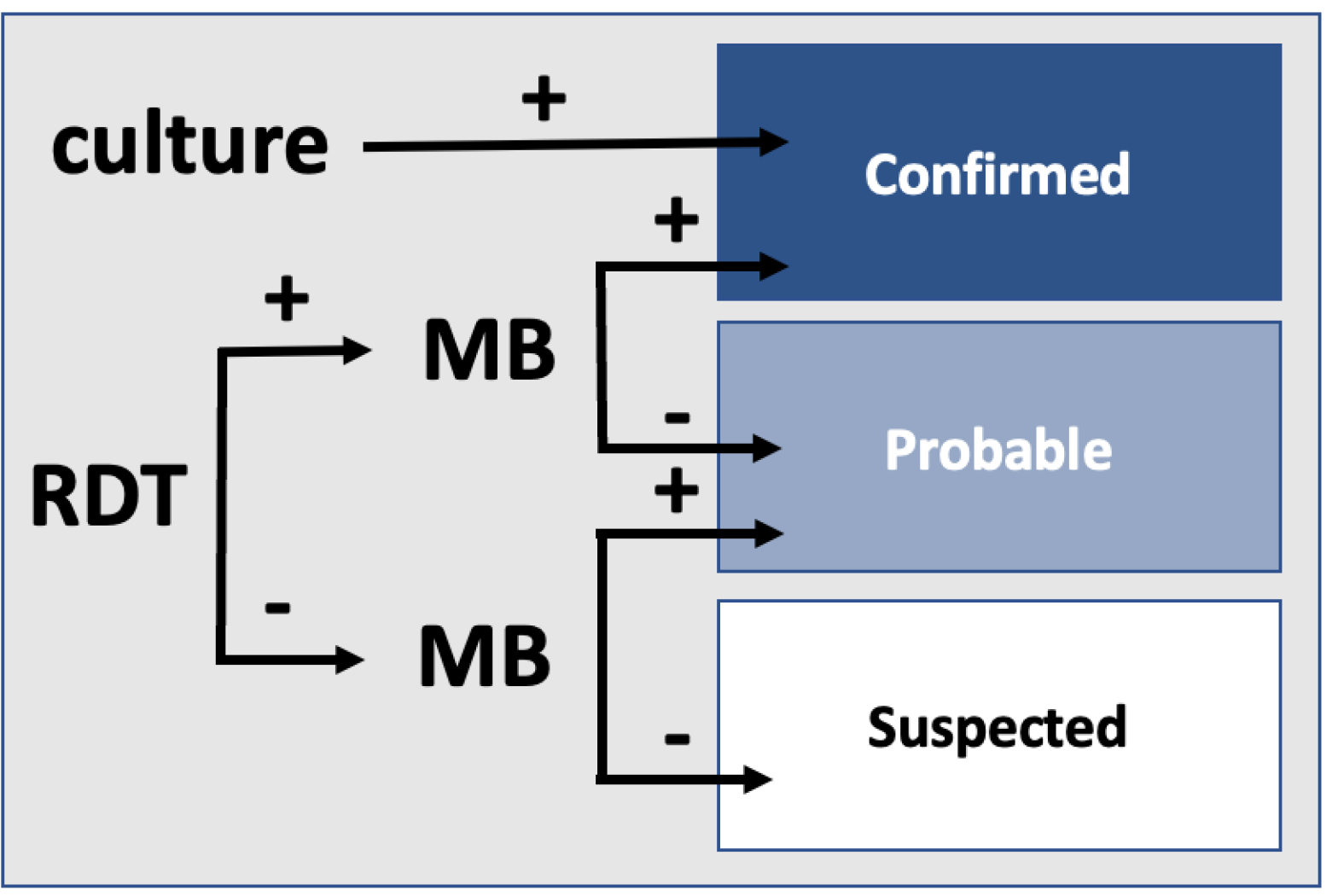

Figure 2. Case classification algorithm: Confirmed cases include cases with positive results for both rapid diagnostic test (RDT) and molecular biology (MB) and/or positive culture, probable have either RDT or MB positive, and suspected have no confirmatory laboratory results.

We develop a latent-class statistical model (13) to estimate the performance of diagnostic tests and the scale of the outbreak from contingency tables describing three tests with up to seven separate diagnostics outcomes (i.e., two single-outcome tests: RDT, culture; plus up to five genes for MB) for 2,136 samples received at the central laboratory for plague (CLP) between August 1 and November 26 2017. The model describes the joint expected distribution of diagnostic outcomes as a function of the prevalence (proportion of $Y$. pestis infections among notified, clinically suspected cases), the sensitivity (probability of positive result if the sample is from a $Y$. pestis-infected person), and specificity (probability of negative result if the sample is from a person that was not infected with $Y$. pestis) of each test. Estimation of model parameters is performed in a Bayesian framework via Markov chain Monte Carlo Sampling (14) under the assumption that culture specificity is $100 \%$. Technical details are provided in Materials and Methods.

We estimate that test specificity was similar between sample types. MB was highly specific (PP: $100 \%$, 95\% credible interval 99-100, BP: 100\%, 98-100), whereas RDT specificity was around $80 \%$ for both PP (82\%, 80-84\%) and BP (85\%, 81-89\%) (Fig 3A,Table S1). Additional analyses including an initially implemented classical Polymerase Chain Reaction protocol (CPCR) confirm that these lacked specificity (PP: 55\%, 52-58\%, BP: 62\%, 56-69\%) (Table S2) and justifies its timely replacement by MB. The latter was the most sensitive test (PP: 80\%, 61-97\%; BP: 95\%, 86-100\%), markedly higher than that of culture (PP: 7\%, 0-23\%; BP: 64\%, 46-85\%) and RDT (PP: 28\%, 18-41\%, BP: 72\%, 61-83\%) (Fig 3B, Table S1). The statistical analysis also provides estimates of the performance of diagnostic tests that would be based on single gene diagnostic outcomes obtained from the qPCR (Table S1). Estimates were robust for 
deviations from model assumptions including the inclusion of the initial CPCR (Table S2), not fixing the specificity of culture to $100 \%$ (Table S4), and the use of a uniform prior on prevalence (Table S5).
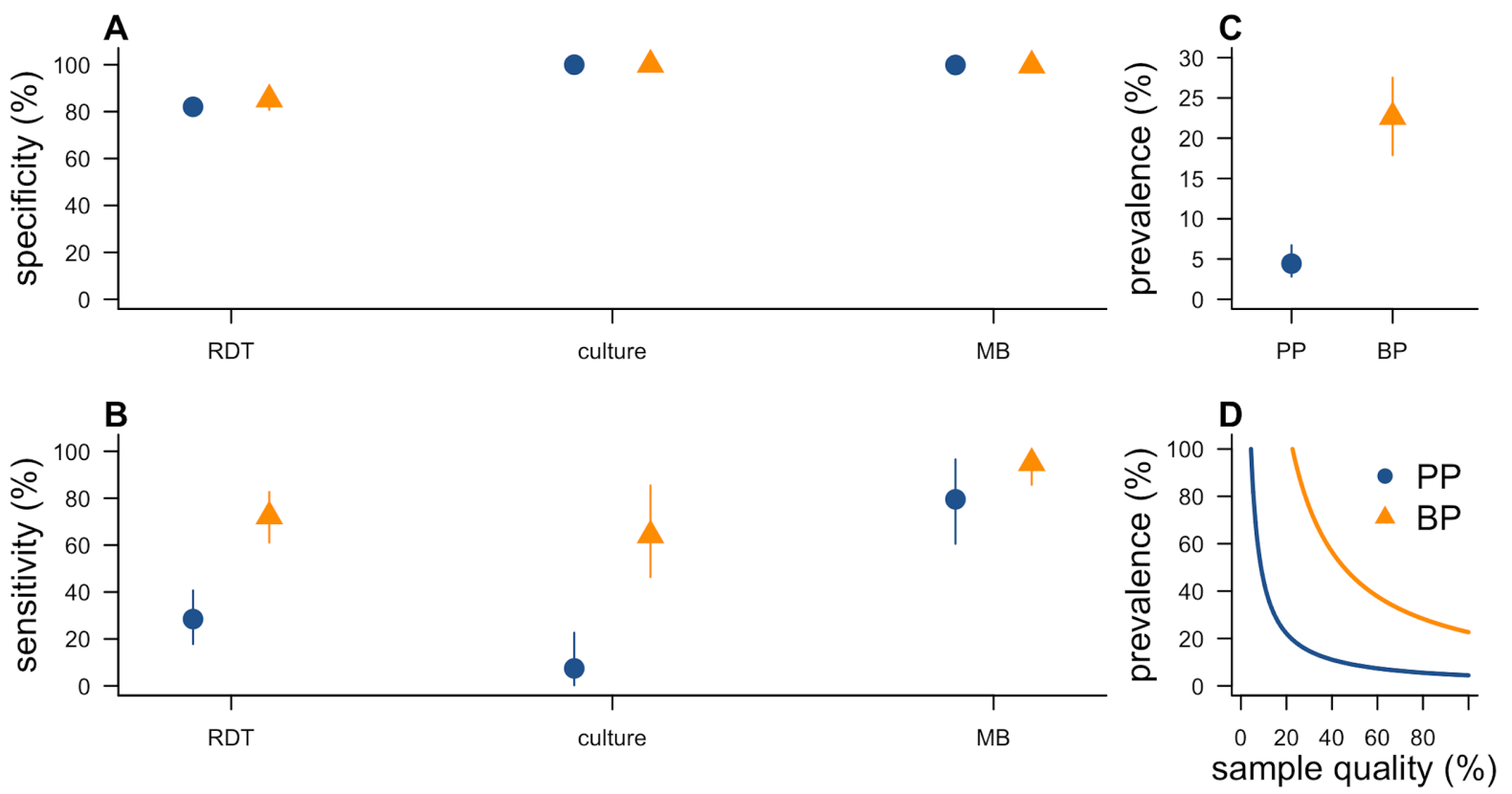

Figure 3. Model estimates of test performance and prevalence. (A) Specificity of each test, with RDT denoting rapid diagnostic test and MB molecular biology. (B) Sensitivity of each test. (C) Prevalence of $Y$. pestis infection among notified cases, under the assumption of perfect sample quality. (D) Relationship between sample quality (i.e., the proportion of samples from infected individuals that contain detectable bacterial material) and estimated prevalence of infection among notified cases. Results are presented by clinical form: pneumonic (PP: blue) and bubonic (BP: orange). The circle/triangle shows the posterior median of the parameter while the lines shows the $95 \%$ Credible Interval.

Under the assumption that samples were of good quality, we estimate that prevalence of infection among notified cases was 4\% (3-7) for PP and 25\% (18-28) for BP (Fig 3C). This corresponds to 78 (50119 ) and 81 (64-98) Y. pestis infections among notified $P P(N=1779)$ and $B P$ cases $(N=357)$, respectively. However, a challenge in diagnosing PP is the risk for samples to be of poor quality, i.e., that samples from a $Y$. pestis-infected individual do not contain detectable bacterial material. If a proportion of samples were of poor quality, estimates for the prevalence of infection would increase (Fig 3D). For example, in the extreme scenario where only $50 \%$ of samples were of good quality, estimates of the prevalence of infection would rise to $9 \%$ (6-13) for PP and 45\% (36-55) for BP. For this analysis we assumed sample quality to affect all tests equally. We also assessed a scenario in which test sensitivities were not fully independent and only the two qPCR gene results were affected by sample quality. This did not improve model fit (Fig S2) and most parameter estimates were robust to departures from the assumption of test independence (Fig S3).

We find that these estimates present good adequacy with the observed data (12), and can accurately reproduce i) the number of notified cases classified as confirmed (PP: 19, 8-47 expected vs 27 observed; BP: $58,37-81$ vs 57) and probable (PP: $356,338-377$ vs 364 , BP: $66,45-87$ vs 66 ) (Fig 1C), ii) the number of notified cases testing positive for RDT, culture, or MB (Fig 1D), and iii) the more detailed contingency table of the different diagnostic outcomes used for inference (Fig 1E). 
lower than the proportion of confirmed or probable cases (Fig 4A). In a scenario of low prevalence, the suboptimal specificity of RDT means that classification for PP based on confirmed or probable cases is characterized by a proportion of false positives (approx. 1-specificity) that is large relative to the prevalence. In contrast, a classification that solely relies on confirmed cases consistently underrepresents the prevalence due to low sensitivity of RDT and culture. For BP, the case classification performs well at any prevalence level, with the true prevalence always falling between the proportion of confirmed and confirmed/probable cases (Fig 4B, Fig S4B).
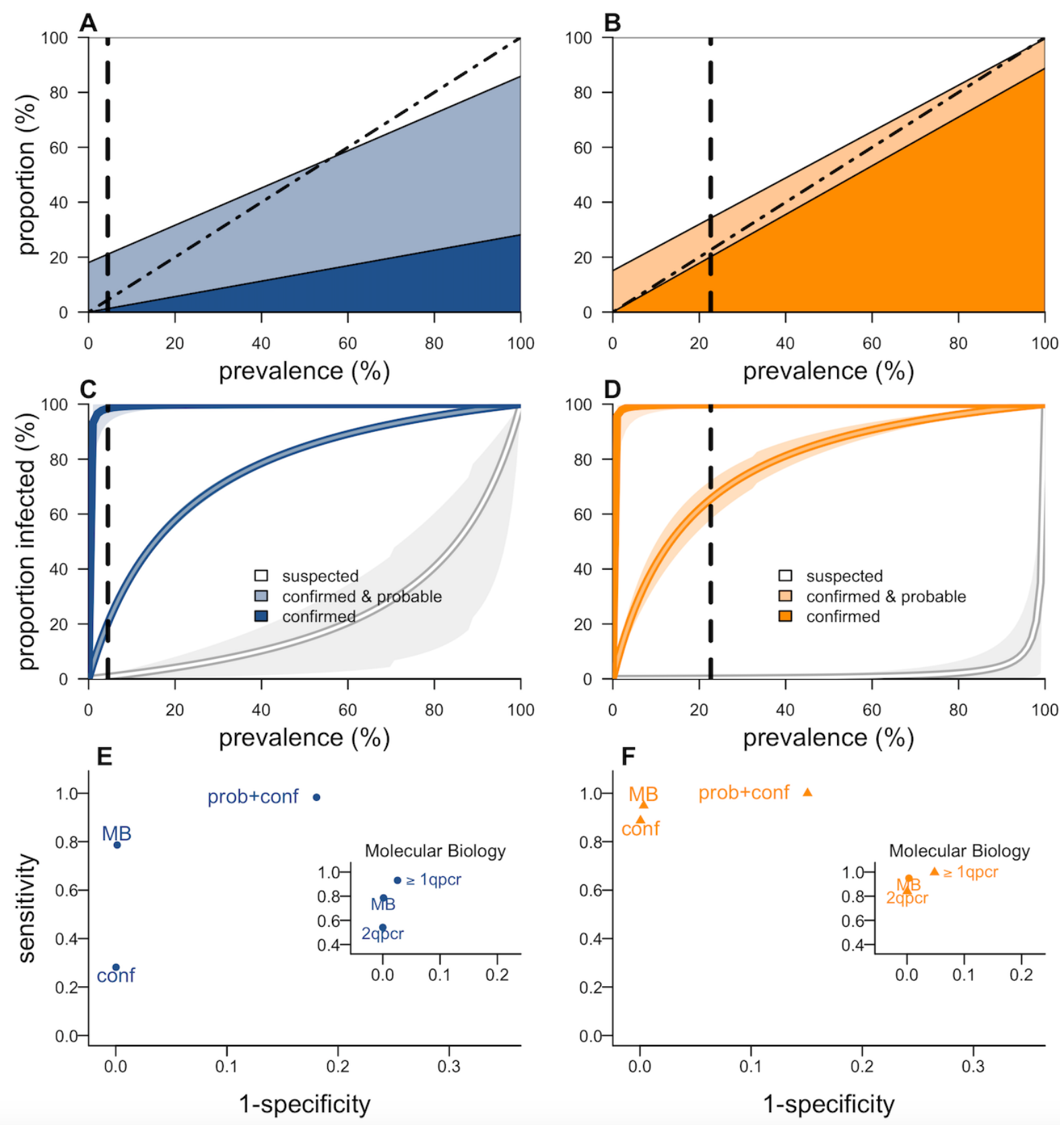

Figure 4. Performance of the case classification system. $(A, B)$ Expected proportion of notified cases classified as confirmed (dark blue or orange), probable (light blue or orange), and suspected (white), as a function of prevalence of infection for PP (A) and BP (B). The dashed vertical line indicates the prevalence among notified cases estimated during the 2017 Madagascar outbreak. The dashed diagonal line corresponds to perfect classification (C,D) Expected proportion of $Y$. pestis infections among cases in the category confirmed, confirmed or probable, and suspected as a function of prevalence of infection for PP (C) and BP (D). (E,F) ROC plots presenting sensitivity versus (1specificity) for a range of possible classification criteria for pneumonic (PP) (E) and bubonic plague (BP) (F) and for simplifications of the MB algorithm for pneumonic (PP) (inset of $\mathrm{E}$ ) and bubonic plague (BP) (inset of F). MB is considered here due to its potential for being considered 
as a classifier by itself. Here conf denotes confirmed and prob probable. Classifications $\geq 1 \mathrm{qpcr}$ and 2 qpcr represent results based on qPCR solely, i.e., in the absence of confirmatory $\mathrm{CPCR}$, with $\geq 1$ qpcr denoting 'at least one gene positive' and 2qpcr 'both genes positive'.

The positive predictive value (PPV) for a category of cases is the proportion of cases of that category that are $Y$. pestis-infected. As expected, we find that the PPV of the confirmed or probable category is strongly impacted by prevalence among notified cases (Fig 4C,D). For example, if the prevalence of PP was $20 \%$, over half of confirmed or probable cases would be expected to be $Y$. pestis-infected. This proportion drops to as little as $22 \%$ (21-24\%) for a prevalence of $5 \%$. This shows that it is critical to avoid overreporting and ensure notified cases meet the clinical case definition. Cases classified as confirmed were, for both clinical forms, almost all Y. pestis-infected (PP: 98\%, 91-100\%; BP: 100\%, 99$100 \%)$, deriving from perfect specificity of culture and the strict criterium requiring both RDT and MB to be positive. We further assess the risk of missing $Y$. pestis-infected cases and predict that 29\% (16$42 \%$ ) of $Y$. pestis-infected PP cases were classified as confirmed and $87 \%$ (73-98) as confirmed or probable. This classification-sensitivity is better for BP with $89 \%$ (81-96\%) of infected cases being confirmed and $100 \%$ (99-100\%) being confirmed or probable. The performance of case classification would be hampered if a substantial proportion of samples was of poor quality (Fig S4).

We can also determine how to revise the classification system to minimize the proportions of false positive (1-specificity) and false negative cases (1-sensitivity) (Fig 4E,F). Best classification for both forms is based on $\mathrm{MB}$, with a proportion of false positive and false negative cases respectively reduced from $2 \%$ to $0 \%(0-0 \%)$ and from $71 \%$ to $20 \%$ (3-40\%) for PP (BP: $0 \%$ to $0 \%, 0-2 \%$ and $11 \%$ to $5 \%, 0$ $14 \%)$ (Fig 4E,F), providing a robust representation of the prevalence (shaded area, Fig 4 A,B).

We then compare the MB algorithm (Fig S1) to simpler alternatives that would not require confirmatory CPCR. We show that the MB algorithm is more sensitive than classification based on qPCR alone using "both genes positive" as a criterium, and more specific than the one using "at least one gene positive" (Fig 4E,F).

Concordance between RDT and MB improved over time among negative MB samples (Fig S5B,D) but decreased among positive MB samples for PP (Fig S5A). We investigate possible changes in RDT performance during the epidemic. We find that RDT specificity increased significantly from 72\% (6976\%) before week 41 to 95\% (93-97\%) afterwards for PP (BP: 71\%, 63-78\% to 98\%, 95-100\%). Sensitivity of RDT was unchanged for BP (73\%, 59- $87 \%$ to $72 \%, 55-88 \%$ ) but decreased for PP (34\%, $16-53 \%$ to $14 \%, 3-30 \%$ ) (Table S3). Earlier and later cut-off times result in a lesser fit (Fig S6). Estimates of RDT specificity for the second part of the outbreak are consistent with those obtained for the subsequent endemic BP season, during which the same batch was used (specificity: 99\%, 96-100\%), and are quite consistent with estimates from earlier evaluations of this test (64\% sensitivity and $93 \%$ specificity based on latent class analysis) (11). The $19 \%$ increase sensitivity estimated in the subsequent BP season (91\%, 84-96\%) suggests that outbreak-specific factors may have indeed hampered RDT and case-classification performance in 2017 (Fig S7).

Lastly, we can use our framework to derive, for each notified case, the probability of $Y$. pestis-infection given their test results (i.e., the positive predictive value). The probability is highest among cases with positive MB (100\%) (Fig S8) or culture (100\%). We then use these estimates, together with the location and timing of cases, to reconstruct the dynamics of spread corrected for spatiotemporal variations in prevalence. Prevalence of $Y$. pestis infections among notified PP cases was threefold (BP: twofold) lower during the outbreak phase (weeks 39-43; when $75 \%$ of notifications occurred) than during the 
initial phase (Fig $5 A, B$ ). Such phenomenon is common when an outbreak receives a lot of attention from authorities, media, and communities, as was the case in 2017. Prevalence of $Y$. pestis infection among notified cases was highest in plague-endemic regions (BP: three-fold higher than Antananarivo), where health-personnel is accustomed to responding to bubonic plague (Fig $5 C, D$ ). Prevalence was lower among children (< five year old) among notified BP cases, but not for PP (Fig 4 $\mathrm{E}, \mathrm{F})$. Correcting for temporal variations in the prevalence, we find that the transmission of $Y$. pestis during this outbreak was less efficient than what was suggested by the analysis of notified cases, particularly for PP: the doubling time in the first 6 weeks was estimated to be 18 rather than 6 days (or 8 based on confirmed/probable) (BP: 24 vs 13 (17)) (Fig 5G,H).
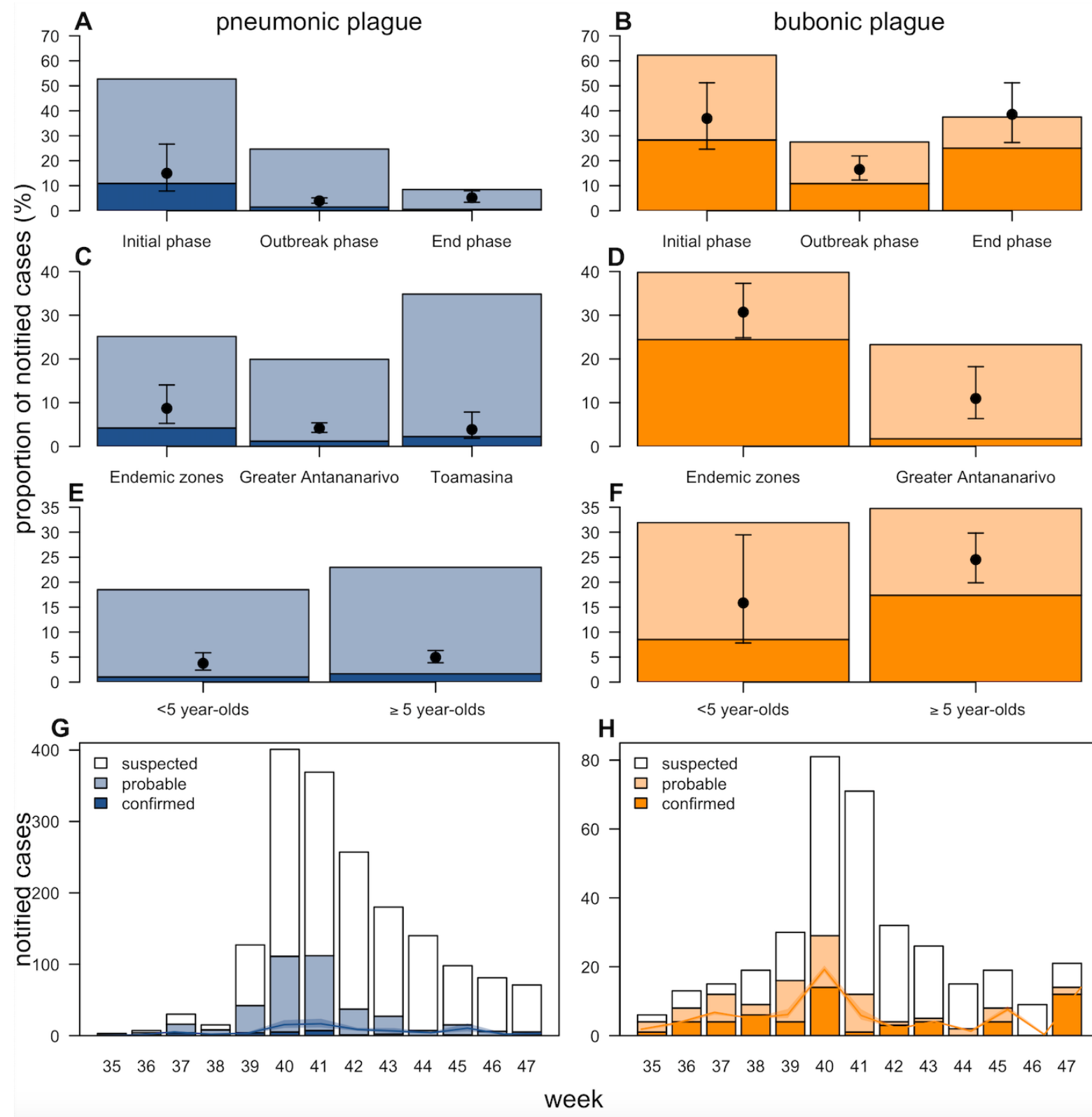

week

Figure 5. Reconstruction of the outbreak by place and time. (A, B) Estimated prevalence of infection among notified cases by time period for pneumonic (PP) (A) and bubonic plague (BP) (B). Here, the initial phase spans weeks 34-38, outbreak phase 39-43, and the end phase 44-48. (C,D) Prevalence estimates by zone for PP (C) and BP (D). No BP cases were notified from Toamasina. (E, F) Prevalence estimates by age for $P P(E)$ and $B P(F) .(G, H)$ Observed notifications vs estimated infections among notified cases for PP $(G)$ and $B P(H)$. The stacked bar plots denote the percentage $(A-F)$ and absolute numbers $(G-H)$ by case classification. 
Assessing the true burden of an outbreak can be difficult in the absence of "gold standard" diagnostic. This can be especially problematic when scarce resources need to be allocated for outbreak control. Here, using plague as a case study, we presented a statistical framework based on latent-class models to parse the results from multiple imperfect diagnostics and assess the true burden of the outbreak. We showed that around one tenth of notified cases were likely to be infected with Yersinia pestis. We showed that, particularly in scenarios with substantial misclassification of cases, poor specificity of some diagnostics can greatly skew case-classification, even if a combination of diagnostic tests is used, and contribute to an overestimation of the true burden of infection. We used estimates of diagnostic test performance together with individual test results to reconstruct epidemiological trends in the proportion of true infections among notified cases and showed that misclassification of cases was highest during the peak of the epidemic and in regions non-endemic to plague. We illustrated the importance of optimized case-classification algorithms, highlighting an overestimation of the transmission potential of the bacteria if based on the, typically used, tally of confirmed and probable cases.

This study highlights challenges inherent to plague diagnostics, particularly those of pneumonic cases. While specificity of test results were similar between bubon and sputum samples, the sensitivity of all diagnostics was substantially lower for sputum samples. This is in line with other respiratory illnesses such as pneumonia (10). Poor quality samples may well result in an underestimation of the true prevalence of infection among notified cases. We assessed how limited sample quality would affect our findings and showed that our general conclusion that the majority of notified cases were not infected with Yersinia pestis is robust to substantial amounts of poor quality samples. We came to the same conclusion if sample quality issues affected some diagnostics more than others.

Classifying cases into confirmed, probable, and suspected is a routine public health effort that gives insight into the extent of the outbreak and an indication of the levels of uncertainty surrounding this. We highlight the importance of accurate classification algorithms and show that, particularly for diseases with non-specific symptoms and high risks of misclassification (e.g. due to raised awareness, or non-familiarity with the disease among public health responders), classification based on tests with poor specificity can result in vast overestimations of the outbreak extent. In the case of the plague outbreak in Madagascar, limited RDT specificity contributed to the majority of probable pneumonic plague cases not to be infected with Yersinia pestis. We showed that the performance of the RDT improved towards the end of the outbreak. Such evolving RDT performance might be explained by the extreme circumstances surrounding this outbreak which may have resulted in changes within laboratories, e.g. due to overworked personnel or, conversely, changes in workflow to increase efficiency and proficiency of sample processing. It might also be due to a change of RDT batch that occurred in week 43. Assessing historical data (11) as well as data from a subsequent outbreak year indeed revealed better RDT test performance than was observed during the first half of the outbreak. The performance of case-classification algorithms may therefore be better during non-outbreak years and care should be taken to uphold this in crisis situations. Apart from upholding test performances, this may also include robust clinical case definitions to prevent large overreporting. Even under better conditions however, the RDT is likely of limited value for case classification when other tools are available. Yet, RDTs are vital for point-of-care diagnostics in peripheral health settings, in particular for BP. Improving RDT performance should therefore be prioritized. Similarly, the inclusion of culture did 
not improve case classification, owing to its limited sensitivity. While the proportion of confirmed cases gave the best indication of the true proportion of infections, a large underestimation of the true burden of infections is expected in scenarios with less overreporting and a higher prevalence among notified cases. The inclusion of culture nevertheless remains fundamental for assessing circulating strains and the antibiotic resistance thereof. In this outbreak, this was particularly relevant as widespread use of prophylactic treatment was observed in response to the large volume of notified cases. The real risks of resistance emergence associated with widespread use are another reason why accurate case classification is important.

Molecular biology (MB) had the best specificity and sensitivity. Especially for BP, adding other diagnostic tests to classify cases does not improve our ability to accurately classify cases. For PP, MB by itself would result in somewhat lower sensitivity than if used in combination with culture and RDT, but the reduced specificity outweighs this benefit. We also assessed whether the MB algorithm (Fig S1) itself could be further improved. While the MB algorithm, particularly for PP, is somewhat less sensitive than the one using "at least one gene positive", we pose that increased specificity should be prioritized in low prevalence scenarios such as the one described here, confirming the relevance of the confirmatory CPCR performed in the MB algorithm.

The integrative framework presented here makes it possible to assess the performance of diagnostics, optimize their use for case classification, and reconstruct the whereabouts of infected cases during outbreaks in situations where no diagnostic gold standard is available. Improved case classification is particularly important for the allocation of scarce resources, for instance by accurately targeting contact tracing efforts and optimizing the impact mobile test facilities. Beyond plague, such analytical framework could be a valuable tool to reduce uncertainties in other infectious disease outbreaks affected by non-optimal diagnostics. This is particularly important when overreporting is likely due to nonspecific symptoms or if mass testing is applied. Above all, the development and availability of highquality diagnostics remains a priority, particularly for pathogens prone to causing explosive outbreaks such as $Y$. pestis.

\section{Materials and Methods}

\section{Background information about plague in Madagascar}

Madagascar accounts for $75 \%$ of plague cases worldwide (5). Health professionals are required to notify all cases clinically suspected of Yersinia pestis infection to the Central Laboratory for Plague (CLP; WHO Collaborating Center) at the Institut Pasteur de Madagascar (IPM), where case notification forms are recorded and biological samples analyzed for laboratory confirmation. Treatment of cases is not contingent on biological confirmation of CLP. Annually, between 200-700, mostly bubonic (BP) (75\%), cases are notified. The majority of these cases occur in the rural central highlands during the country's plague season (October-April). Occasional small outbreaks of pneumonic plague (PP) were recorded in 1997, 2011, and 2015 in rural areas (15-18) and in 2004 in one commune of the county's capital city Antananarivo.

Between August and November of 2017, the country experienced an outbreak that, with 2,414 notified cases, was much larger than regular plague seasons and presented with an unusual proportion of cases with clinically suspected PP. 
Samples from clinically suspected cases $(2,414)$ were sent to the Central Laboratory for Plague (CLP) at the Institut Pasteur de Madagascar for diagnostic testing. Treatment of suspected cases was not contingent on biological results. Biological samples were taken from cases presenting at health care settings with symptoms consistent with plague (i.e., for BP: presence of an isolated, painful adenopathy, for PP: cough (< 5 days), bloody sputum, chest pain with fever) (3). There was no formal clinical case definition that needed to be satisfied for patients to be tested. Samples included bubo aspirates from BP and secondary PP, sputum samples for PP, and liver and/or lung aspirates from deceased cases. All samples were tested for fraction 1 (F1) capsular antigen using a rapid diagnostic test (RDT) (11). Initially, molecular biology was performed using conventional PCR (CPCR) targeting the pla gene on all samples. Due to low specificity of the CPCR, this test was abandoned on November 3 and replaced by real time quantitative PCR (qPCR) targeting pla and caf1 genes. If both genes tested positive, a sample was considered positive for molecular biology. Samples with discordant or inconclusive qPCR results were verified using confirmatory CPCR on pla, caf1, and inv 1100 genes, with protocols improved to reach better specificity. They were considered positive upon positive results for the inv $v_{1100}$ gene and/or for both pla and caf1 genes (see Fig S1 for decision tree) (19). All samples received before November 3 were re-tested using the molecular biology protocols (November December 2017). In addition, culture was performed on all samples with positive RDT (20). PP samples from non-endemic regions received between September 11 and October 3 were cultured irrespective of RDT result. No serological testing was performed during the outbreak.

As per WHO guidelines, cases were classified based on their diagnostic test results as confirmed if culture and/or both RDT and MB were positive, probable upon positive results for either MB or RDT, and suspected otherwise (Fig 2). Initial CPCR results were not considered for case classification. Culture is often regarded as a gold standard given its perfect specificity, yet lacks sensitivity. Culture sensitivity may have been particularly challenged during this outbreak, as a result of widespread prophylactic antibiotic use (21).

\section{Estimating diagnostic test performances and burden of infection}

We develop a statistical model based on latent class methods to estimate test performances and burden of infection among the population of notified cases $(N)$. Here, we distinguish diagnostic outcomes as the raw outcomes of performed qPCRs (i.e., gene-specific outcomes) and the composite result of the confirmatory CPCR (Fig S1). The composite of these make up the result of a diagnostic test. For each notified case (i) dichotomous results $\left(y_{i j}\right)$ are available for up to $J$ diagnostic outcomes $(j)$ (i.e., one for each of RDT and culture, and up to three for MB: two for qPCR (pla, caf1) and one for confirmatory CPCR ( $p l a, c a f 1$, inv $v_{1100}$ ), with $y_{i j}=1$ denoting a positive and $y_{i j}=0$ a negative result. The infection status of case $i$ is denoted $d_{i}(=1$ if infected and 0 otherwise). The sensitivity and specificity of diagnostic outcome $j$ are denoted $\left.S_{j}=P\left(Y_{j}=1 \mid D=1\right)\right)$ and $C_{j}=P\left(Y_{j}=0 \mid D=0\right)$, respectively.

Here, we calculate the contribution to the likelihood of the different diagnostic outcomes. Test specific sensitivities and specificities are then calculated from the characteristics of the diagnostic outcomes that make up a specific test (MB in particular). We first discuss the likelihood for those diagnostic outcomes that are performed irrespective of other diagnostic outcomes (RDT, qPCR), followed by those that are performed conditional on other diagnostic outcomes (culture and confirmatory CPCR). 
Contribution to the likelihood of RDT, qPCR (pla, caf1): We first calculate the contribution to the likelihood of diagnostic outcomes that are performed irrespective of other diagnostic outcomes, namely RDT, qPCR (pla and caf1) (indexed in eq. 1 as $1 \ldots U$ ). If the infection status of a case was known, two conditional probabilities would have to be considered:

- Conditional on being infected by plague and given model parameters $\theta$, the joint probability of test results for case $i$ is

$$
P\left(\left\{y_{i j}\right\}_{j=1 \ldots U} \mid \theta, d_{i}=1\right)=\prod_{j=1}^{U} S_{j}^{y_{i j}}\left(1-S_{j}\right)^{1-y_{i j}}
$$

- Conditional on not being infected by plague and given model parameters $\theta$, this probability becomes:

$$
P\left(\left\{y_{i j}\right\}_{j=1 \ldots U} \mid \theta, d_{i}=0\right)=\prod_{j=1}^{U} C_{j}^{1-y_{i j}}\left(1-C_{j}\right)^{y_{i j}}
$$

In practice, the infection status of a case is unknown, and we therefore work on the unconditional joint probability of diagnostic outcomes for case $i$ that integrates over the different possibilities:

$$
\begin{gathered}
P\left(\left\{y_{i j}\right\}_{j=1 \ldots U} \mid \theta\right)=P\left(d_{i}=1\right) P\left(\left\{y_{i j}\right\}_{j=1 \ldots U} \mid \theta, d_{i}=1\right)+P\left(d_{i}=0\right) P\left(\left\{y_{i j}\right\}_{j=1 \ldots U} \mid \theta, d_{i}=0\right) \\
P\left(\left\{y_{i j}\right\}_{j=1 \ldots U} \mid \theta\right)=\pi \prod_{j=1}^{U} S_{j}^{y_{i j}}\left(1-S_{j}\right)^{1-y_{i j}}+(1-\pi) \prod_{j=1}^{U} C_{j}^{1-y_{i j}}\left(1-C_{j}\right)^{y_{i j}}
\end{gathered}
$$

where $\pi$ is the prevalence of plague infection among notified cases.

While RDT, qPCR ( $p$ la and caf1) were performed independent of other results, culture was done based on RDT outcome, period and zone, and confirmatory CPCR (pla, caf1, inv ${ }_{1100}$ ) was performed only if qPCR was inconclusive. We need to integrate such conditioning in our analysis to avoid biases.

Contribution to the likelihood of culture: For PP samples received from a non-endemic region between September 11 and October 3, culture was performed irrespective of RDT results and we therefore use the formulation described above. For all other samples, culture was performed only if RDT was positive. Hence, the conditional probability for these individuals to obtain a culture result $\left(y_{\text {icult }}\right)$ is

$$
\begin{aligned}
\mathrm{P}\left(\mathrm{y}_{\mathrm{i} \text { cult }} \mid y_{i_{R D T}}=\right. & 1, \theta) \\
& =\pi \frac{S_{\text {cult }}^{y_{\text {cult }}}\left(1-S_{\text {cult }}\right)^{1-y_{\text {cult }}} S_{R D T}}{P\left(y_{\text {iRDT }}=1\right)} \\
& +(1-\pi) \frac{C_{\text {cult }}^{1-y_{\text {cult }}}\left(1-C_{\text {cult }}\right)^{y_{\text {cult }}}\left(1-C_{R D T}\right)}{P\left(y_{i R D T}=1\right)} .
\end{aligned}
$$


where PPV is

$$
P P V_{R D T}=\frac{\pi S_{R D T}}{\pi S_{R D T}+(1-\pi)\left(1-C_{R D T}\right)}
$$

371

which can also be expressed in terms of the positive predictive value (PPV, the proportion infected among individuals with a positive test result) of RDT

$$
P P V_{R D T} S_{\text {cult }}^{y_{\text {cult }}}\left(1-S_{\text {cult }}\right)^{1-y_{\text {cult }}}+\left(1-P P V_{R D T}\right) C_{\text {cult }}^{1-y_{\text {cult }}}\left(1-C_{\text {cult }}\right)^{y_{\text {cult }}}
$$

Results from culture that did not adhere to this conditioning (PP:283, BP:60) were not included in the analysis as the reason for this additional testing cannot be traced back but was likely non-random and affected by other test results.

Contribution to the likelihood of CPCR (pla, caf1, and inv $v_{1100}$ ): Test results for MB were a composite of up to five diagnostic outcomes (Fig 2). Confirmatory CPCR for genes 1 to $k$ were performed conditional on discordant qPCR results. The composite result of this test was included in the model.

$$
\begin{aligned}
& \mathrm{P}\left(\mathrm{y}_{\text {iconfPCR, }} \mid Y_{i_{q P C R_{p l a}}} \neq Y_{i_{q P C R_{c a f 1}}}, \theta\right)= \\
& \pi \frac{S_{\text {conf } P C R}^{y_{\text {conf } P C R}}\left(1-S_{\text {conf } P C R}\right)^{1-y_{\text {conf } P C R}} *\left(S_{\text {pla }} *\left(1-S_{\text {caf } 1}\right)+\left(1-S_{\text {pla }}\right) * S_{\text {caf } 1}\right)}{P\left(Y_{\text {i } q P C R_{p l a}} \neq Y_{i q P C R_{\text {caf } 1}}\right)}+ \\
& (1-\pi) \frac{C_{\text {confPRC }}^{1-y_{\text {conf } P C R}}\left(1-C_{\text {conf } P C R}\right)^{y_{c o n f P C R}} *\left(C_{p l a} *\left(1-C_{c a f 1}\right)+\left(1-C_{p l a}\right) * C_{c a f 1}\right)}{P\left(Y_{i q P C R_{p l a}} \neq Y_{i q P C R_{c a f} 1}\right)} .
\end{aligned}
$$

$$
\prod_{i: Y_{i}} \prod_{q P C R_{p l a} \neq Y_{i q P C R_{C a f 1}}} \mathrm{P}\left(\mathrm{y}_{\text {iconfPCR }_{\text {pla }},}, Y_{i_{q P C R_{p l a}}} \neq Y_{i_{q P C R_{\text {caf } 1}}}\right)
$$

Inference: Parameter estimation was done in a Bayesian setting using a Bayesian Metropolis-Hastings Markov chain Monte Carlo (MCMC) approach (14). We utilized a weakly informative Beta-distributed prior for prevalence (shape $=1$, scale $=2$ ) (i.e., chance of prevalence being below $50 \%$ is twice as high as above), based on estimates of prevalence from previous BP outbreaks (22). To confirm the robustness of the results to the choice of priors on prevalence, the MCMC was also performed with a 
uniform prior between 0 and 1 (Table S4). For specificities of tests associated with MB, Beta-distributed priors were used with means of $95 \%$ (shape $=12.7$, scale $=0.67$ ), based on verifications done in the IPM laboratories prior to implementation. The specificity of culture was fixed at $100 \%$. For all other parameters, we used uniform priors between 0 and 1 (i.e., for all sensitivities as well as the specificity of RDT). Metropolis-Hastings updates were performed on a natural scale with step sizes adjusted such to obtain an acceptance probability between 10 and 50\% (14). Traces of the MCMC were plotted per parameter and convergence was assessed visually (Fig S9 and S10).

Assuming imperfect sample quality: Collection of good quality samples is challenging and might be affected by prophylactic treatment, preservation techniques, and the delays between symptom onset and sample testing at CLP. The prevalence of infection is related to the prevalence of detectable bacterial material in the collected samples $(\tau)$ such that $\tau=\rho \pi$, where $\rho$ is the probability of good sample quality given a truly infected individual. Accounting for $\rho$ in eqn. (3) gives

$$
\begin{gathered}
\mathrm{P}\left(\left\{y_{i j}\right\}_{j=1 \ldots J}\right)= \\
\pi \prod_{j=1}^{J}\left(\rho\left(S_{j}^{y_{j}}\left(1-S_{j}\right)^{1-y_{j}}\right)+(1-\rho) C_{j}^{1-y_{j}}\left(1-C_{j}\right)^{y_{j}}\right)+ \\
(1-\pi) \prod_{j=1}^{J} C_{j}^{1-y_{j}}\left(1-C_{j}\right)^{y_{j}} .
\end{gathered}
$$

Here $S_{\mathrm{j}}$ and $C_{\mathrm{j}}$ denote the absolute sensitivity and specificity, i.e., assuming the sample is of good quality. The definition of $\rho$ implies that all tests are equally affected by factors reducing sample quality.

Assuming dependence between qPCR results: While in the above calculations test results are assumed independent of each other, in practice this may not always be true. Notably, as results from both genes assessed by qPCR are performed in the same assay, possible contaminations or technical problems might affect both test outcomes concurrently. To assess the sensitivity of our results to departures from the assumption of independence, we adjusted the contribution of the outcomes qPCR pla and qPCR caf to the likelihood (eqn. 8) to reflect a larger likelihood of concordance between both diagnostic outcomes

$$
P\left(\left\{y_{1}, y_{2},\right\} \mid \theta, d_{i}=1\right)=S_{1}^{y_{1}} * S_{2}^{y_{2}}\left(1-S_{1}\right)^{1-y_{1}}\left(1-S_{2}\right)^{1-y_{2}}+(-1)^{y_{1}-y_{2}}\left(\operatorname{cov}_{12} \mid d=1\right)
$$

Here, indices 1 and 2 refer to QPCR pla and caf respectively and cov denotes the pairwise covariance between diagnostic outcomes (23). We assess whether the inclusion of a covariance factor affected the fit to the data, using DIC as an indicator of fit (24), and whether estimated test characteristics were robust to departures from assumptions of independence.

Model fit did not improve upon the inclusion of a covariance factor (Fig S2) and parameter estimates were relatively robust: Prevalence of PP was insensitive to the existence of correlations between these tests (4\% vs 6\%) (Fig S3). Prevalence estimates of BP increased for high levels of correlation (35\% vs 
$23 \%)$, which came with increased RDT specificity (97\% vs $85 \%$ ) and reduced sensitivity of culture (41\%

412 vs $64 \%)$.

Over the course of the outbreak: Changes in concordance between RDT and MB were observed towards the end of the outbreak (Fig S5). To test whether a change in RDT-test performance could explain this observation, we re-ran the inference routine allowing RDT to have different test characteristics before and after a predetermined cut-off week (i.e. essentially treating RDT before and after the cut-off point as distinct tests). We used different cut-off weeks (38-43), where the week denotes, as elsewhere in the manuscript, the date of symptom onset of the cases. The model with the cut-off week that yielded the highest likelihood was then compared to the baseline model (i.e., assuming no change in RDT performance) using DIC to test whether the change in RDT-performance resulted in an improved model fit. We did not examine changes in other tests because i) CPCR was terminated halfway through the outbreak, ii) culture only yielded few positive samples, and iii) MB was performed (in retrospect) at the end of the outbreak.

Between outbreaks: We compared results from the outbreak year (2017-2018) with those from the subsequent plague season (2018-2019). Between August 172018 and April 7 2019, 261 (46 PP, 211 $B P, 4$ unknown form) cases were reported to the CLP. All samples were analyzed using MB, RDT, and culture, with the same protocols as were used during the 2017 outbreak year. Among sputum samples for PP ( $n=25), 22$ were negative for both MB and RDT. Others were positive for either or both tests (1 $\mathrm{MB}+\& \mathrm{RDT}+, 1 \mathrm{MB}-$ \& RDT+, $1 \mathrm{MB}+$ \& RDT-), with those positive for MB confirmed by culture. Among bubon aspirates for BP $(n=194), 174$ were concordant between MB and RDT ( 94 positive, 80 negative). Given the low number of positive sputum samples for PP (1 confirmed, 2 probable, 22 suspected), we only analyzed BP samples from this season. Inference was similar to that on samples from 2017 but since culture was performed on all samples, no conditioning was needed when assessing the contribution of culture on the joint likelihood. In addition, due to high concordance between both genes used for qPCR, few conditional PCRs were performed. We thus did not estimate the performance of conditional PCRs during this season.

\section{Outbreak reconstruction}

We derived the probability of $Y$. pestis infection for each notified case based on the positive predictive value associated with their results and assuming the medians of the estimated prevalence, sensitivity, and specificity (see eq 6.). The sum of all PPVs denotes the expected number of true infections among notified cases. We used this relationship to reconstruct the number of expected infections by subgroup. We divided the notified cases according to the following categories: i) by period, distinguishing the initial phase (weeks 34-38), the outbreak phase (weeks 39-43), and the end phase (weeks 44-48), ii) by week, iii) by zone, distinguishing endemic zones (plague-endemic districts (25) apart from greater Antananarivo), greater Antananarivo (urban community of Antananarivo and the three neighboring districts), and Toamasina district, and iv) by age group (below and above 5 years of age). Using these, we estimated the prevalence and exact binomial 95\% confidence interval of infection among notified cases by subgroup. 
All analyses have been performed in $\mathrm{R}(26)$. MCMC results have been processed using the coda (27) and BayesianTools packages (28).

\section{Acknowledgments}

The authors would like to thank for their continuous support during the epidemic the other team members of the IPM laboratories (from the Virology, Experimental Bacteriology, Infectious Diseases Immunology, Malaria Research Units, the Clinical Biology Laboratory, and the Hygiene Food and Environment Laboratory), members of supporting research units at IP Paris (CIBU, Epidemiology of Emerging Infectious Diseases Unit, Molecular Genetics of RNA Viruses Unit), experts deployed through the Global Outbreak Alert Response Network (GOARN) and all the colleagues who were instrumental in improving and implementing epidemiological and laboratory surveillance, and supported the epidemic response efforts. MM, RR, and FMR acknowledge financial support from Wellcome Trust/ Department of International Development (Grant 211309/Z/18/Z).

\section{References}

1. C. Wirsing von König, Pertussis diagnostics: overview and impact of immunization. Expert Review of Vaccines. 13, 1167-1174 (2014).

2. M. C. Brouwer, A. R. Tunkel, D. van de Beek, Epidemiology, diagnosis, and antimicrobial treatment of acute bacterial meningitis. Clin. Microbiol. Rev. 23, 467-492 (2010).

3. R. D. Perry, J. D. Fetherston, Yersinia pestis--etiologic agent of plague. Clin. Microbiol. Rev. 10, 3566 (1997).

4. J. L. Kool, R. A. Weinstein, Risk of person-to-person transmission of pneumonic plague. Clinical Infectious Diseases. 40, 1166-1172 (2005).

5. World Health Organization, Plague around the world, 2010-2015. Wkly Epidemiol Rec. 91, 89-93 (2016).

6. C. A. Nelson et al., Antimicrobial treatment of human plague: a systematic review of the literature on individual cases, 1937-2019. Clinical Infectious Diseases. 70, S3-S10 (2020).

7. A. P. Salam, A. Rojek, E. Cai, M. Raberahona, P. Horby, Deaths Associated with Pneumonic Plague, 1946-2017. Emerg. Infect. Dis. 26, 2432-2434 (2020).

8. E. Bertherat et al., Lessons learned about pneumonic plague diagnosis from two outbreaks, Democratic Republic of the Congo. Emerg. Infect. Dis. 17, 778-784 (2011).

9. A. P. Salam et al., Factors influencing atypical clinical presentations during the 2017 Madagascar pneumonic plague outbreak: a prospective cohort study. Am. J. Trop. Med. Hyg. 102, 1309-1315 (2020).

10. S. Ewig, M. Schlochtermeier, N. Goïke, M. S. Niederman, Applying sputum as a diagnostic tool in pneumonia: limited yield, minimal impact on treatment decisions. Chest. 121, 1486-1492 (2002).

11. S. Chanteau et al., Development and testing of a rapid diagnostic test for bubonic and pneumonic plague. The Lancet. 361, 211-216 (2003). 
12. R. Randremanana et al., Epidemiological characteristics of urban plague epidemic in Madagascar, August-November 2017: an outbreak report. Lancet Infect.Dis. 19, 537-545 (2019).

13. W. J. Rogan, B. Gladen, Estimating prevalence from the results of a screening test. Am. J. Epidemiol. 107, 71-76 (1978).

14. W. R. Gilks, S. Richardson, D. Spiegelhalter, Markov chain Monte Carlo in practice (Chapman and Hall/CRC, , 1995).

15. V. Richard et al., Pneumonic plague outbreak, Northern Madagascar, 2011. Emerg. Infect. Dis. 21, 8-15 (2015).

16. B. Ramasindrazana et al., Pneumonic Plague Transmission, Moramanga, Madagascar, 2015. Emerg. Infect. Dis. 23, 521-524 (2017).

17. M. Ratsitorahina, S. Chanteau, L. Rahalison, L. Ratsifasoamanana, P. Boisier, Epidemiological and diagnostic aspects of the outbreak of pneumonic plague in Madagascar. The Lancet. 355, 111-113 (2000).

18. S. Rakotosamimanana et al., A decade of plague in Madagascar: A description of two hotspot districts. BMC Public Health. 21, 1-9 (2021).

19. C. Loiez et al., Detection of Yersinia pestis in sputum by real-time PCR. J. Clin. Microbiol. 41, 48734875 (2003).

20. B. Rasoamanana, L. Rahalison, C. Raharimanana, S. Chanteau, Comparison of Yersinia CIN agar and mouse inoculation assay for the diagnosis of plague. Trans. R. Soc. Trop. Med. Hyg. 90, 651 (1996).

21. A. P. Salam et al., Factors Influencing Atypical Clinical Presentations during the 2017 Madagascar Pneumonic Plague Outbreak: A Prospective Cohort Study. Am. J. Trop. Med. Hyg. 102, 1309-1315 (2020).

22. V. Andrianaivoarimanana et al., Trends of Human Plague, Madagascar, 1998-2016. Emerg. Infect. Dis. 25, 220-228 (2019).

23. D. Berkvens, N. Speybroeck, N. Praet, A. Adel, E. Lesaffre, Estimating disease prevalence in a Bayesian framework using probabilistic constraints. Epidemiology., 145-153 (2006).

24. K. P. Burnham, D. R. Anderson, Model selection and multimodel inference: a practical information-theoretic approach (Springer Science \& Business Media, , 2003).

25. V. Andrianaivoarimanana et al., Understanding the persistence of plague foci in Madagascar. PLoS Neglected Tropical Diseases. 7, e2382 (2013).

26. R Core Team, R: A Language and Environment for Statistical Computing. (2021).

27. M. Plummer, N. Best, K. Cowles, K. Vines, CODA: Convergence diagnosis and output analysis for MCMC. $R$ News. 6, 7-11 (2006). 
521 28. F. Hartig, F. Minunno, S. Paul, D. Cameron, T. Ott, BayesianTools: General-purpose MCMC and 522 SMC samplers and tools for Bayesian statistics. R Package Version 0.1. 6(2019). 


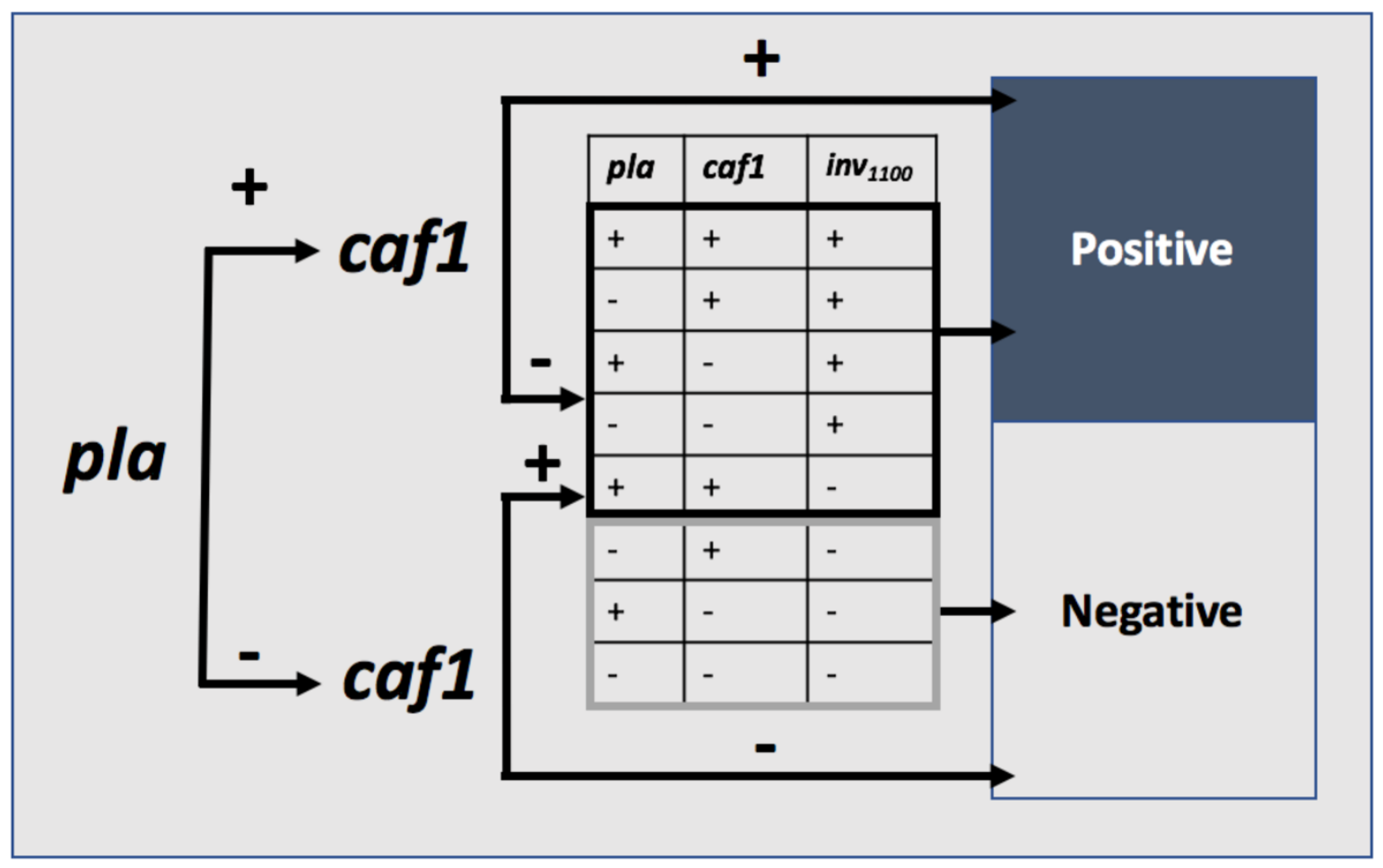

Fig S1. Molecular Biology (MB) algorithm. All samples were tested for the presence of pla and caf1 genes using qPCR. In case of discordance between two genes, an additional conventional PCR (box) targeting genes pla, caf1, and inv 1100 was performed. Samples were then considered positive upon positive results for $i v_{1100}$ and/or positive results for both pla and caf1. 


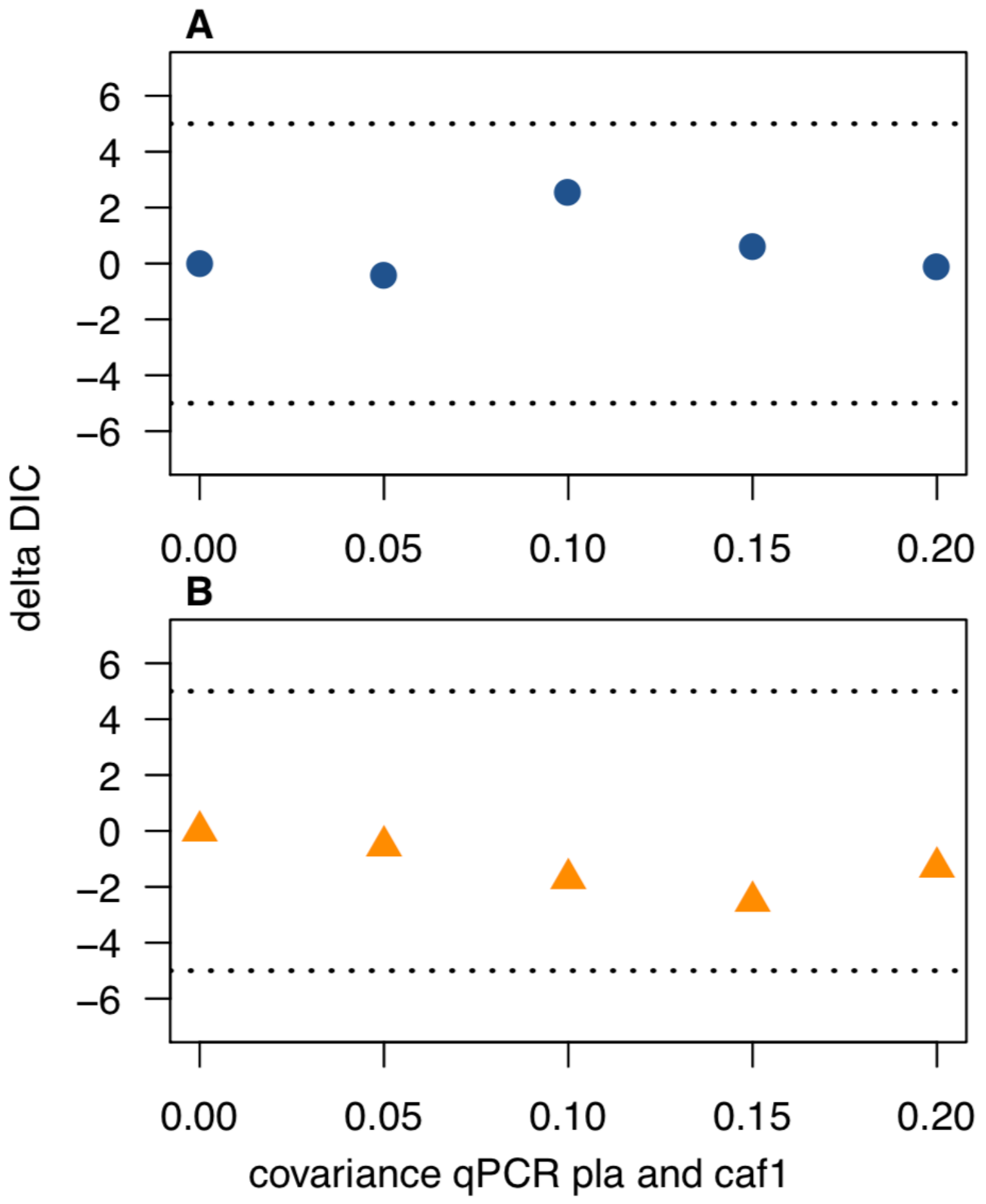

Fig S2. Model fit as a function of covariance between qPCR pla $_{\text {and }}$ qPCR caf1 $_{1}$ sensitivities for pneumonic forms (A) and bubonic forms (B). DIC is the deviance information criterium, with lower values indicating a better model fit. Delta DIC denotes the difference between the baseline model (no covariance) and the models with increasing levels of covariance. Differences larger than 5 DIC (dotted line) are generally considered to depict significant improvement in model fit (24) . 


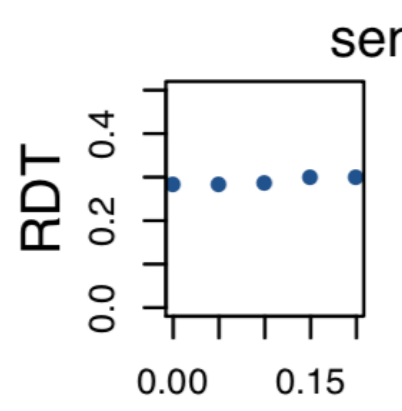

sensitivity
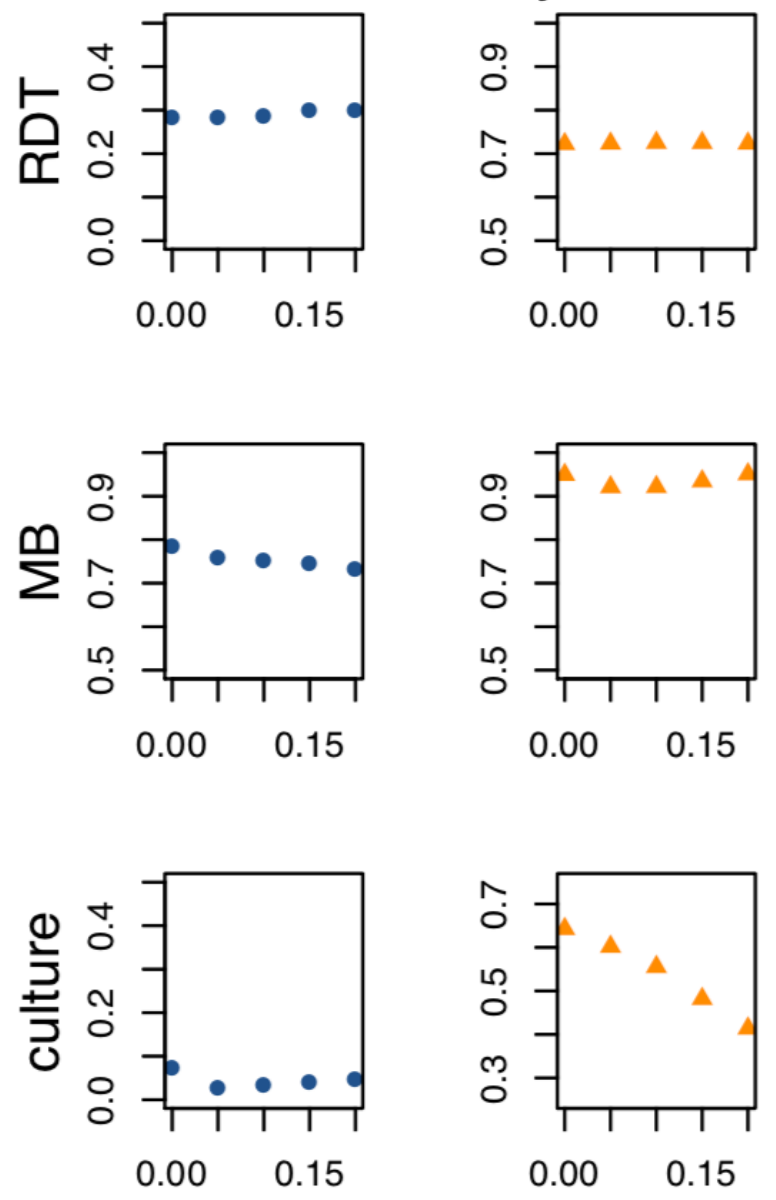

536

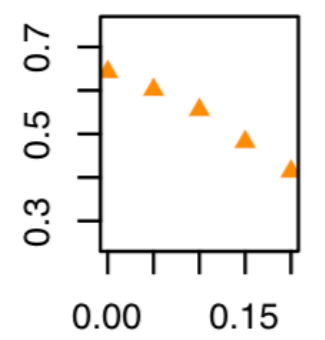

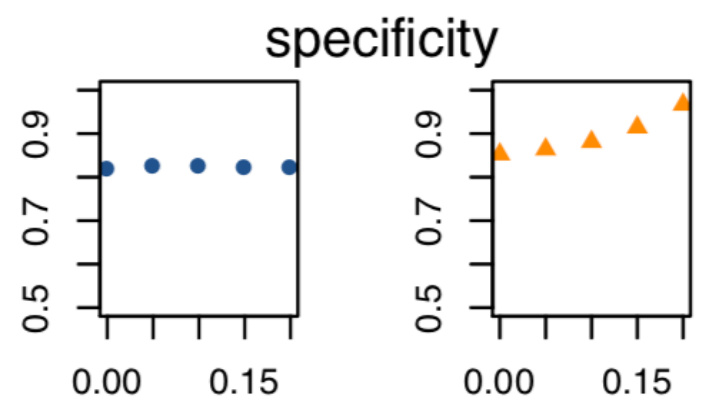
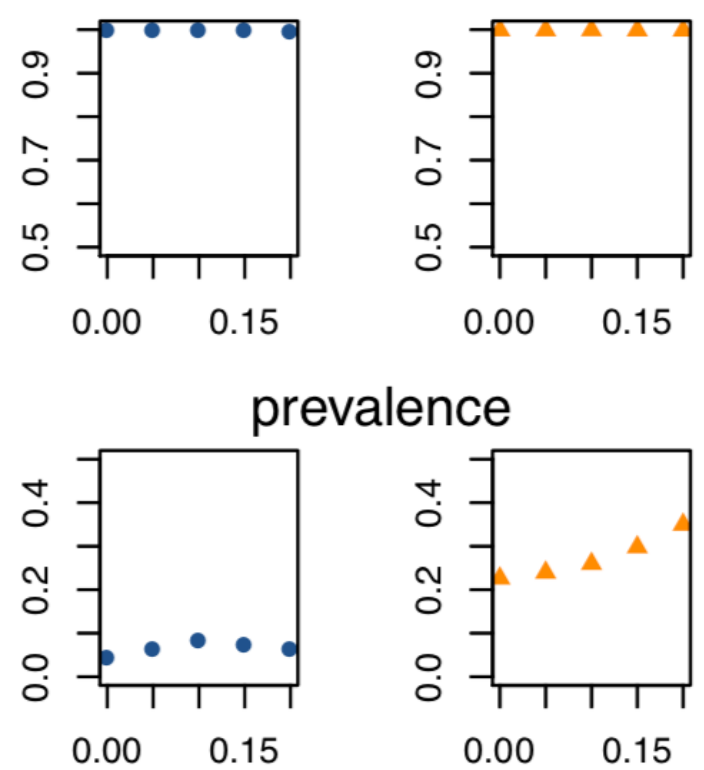

prevalence

covariance

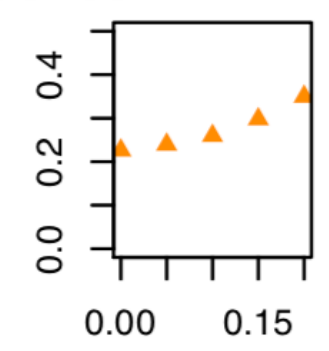



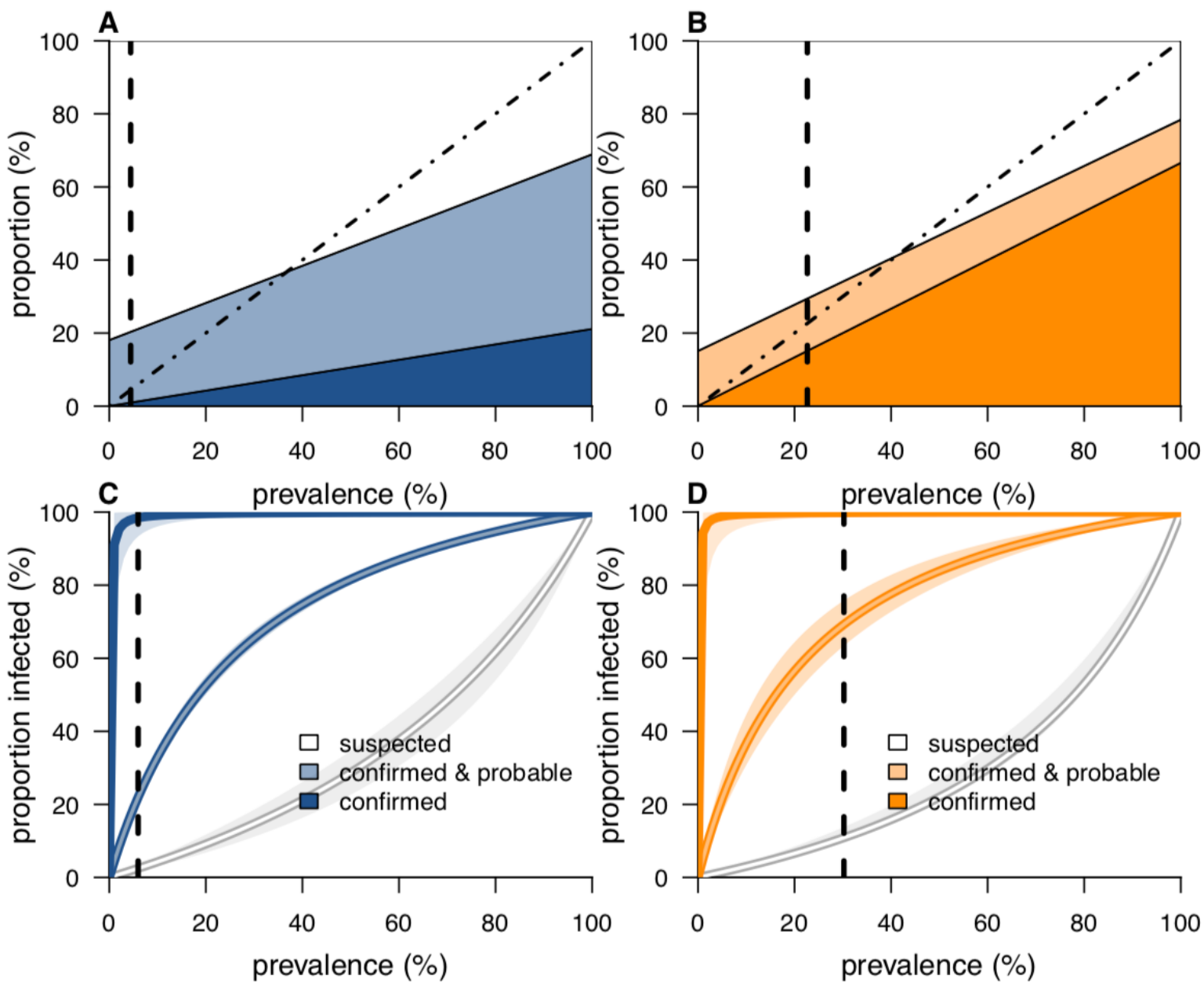

Fig S4. Performance of the case classification system assuming a sample quality of $\mathbf{7 5 \%}$. $(A, B)$ Expected proportion of notified cases classified as confirmed (dark blue or orange), probable (light blue or orange), and suspected (white), as a function of prevalence of infection for PP (A) and BP (B). The dashed vertical line indicates the prevalence estimated during the 2017 Madagascar outbreak. (C,D) Expected proportion of $Y$. pestis infections among cases in the category confirmed, confirmed or probable, and suspected as a function of prevalence of infection for PP (C) and BP (D). 

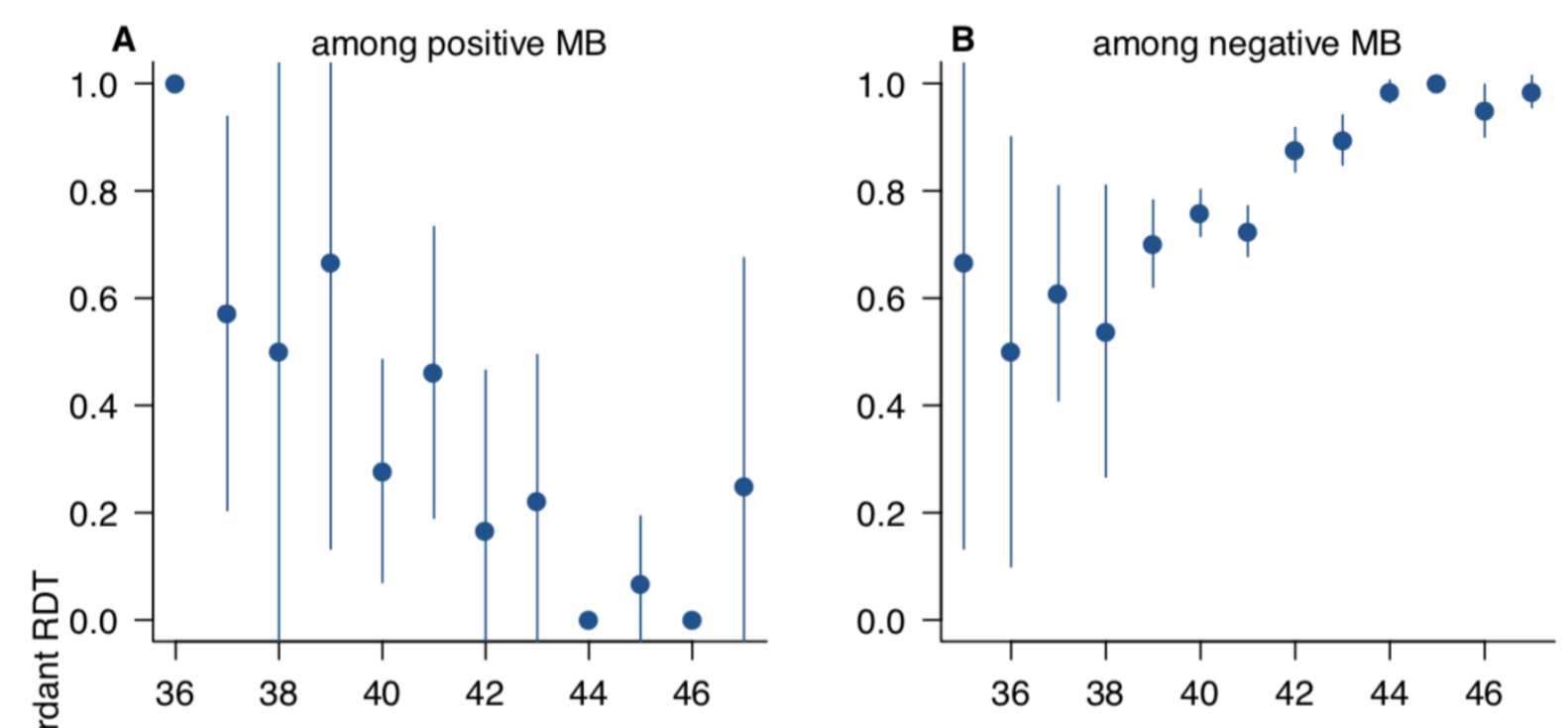
Fig S5. RDT vs MB concordance over time. Observed number of samples among positive $(A, C)$ and negative molecular
biology $(M B)(B, D)$ with concordant results for the rapid diagnostic test (RDT). Pneumonic samples (blue: $A, B)$ and bubonic samples (orange: $C, D)$. 


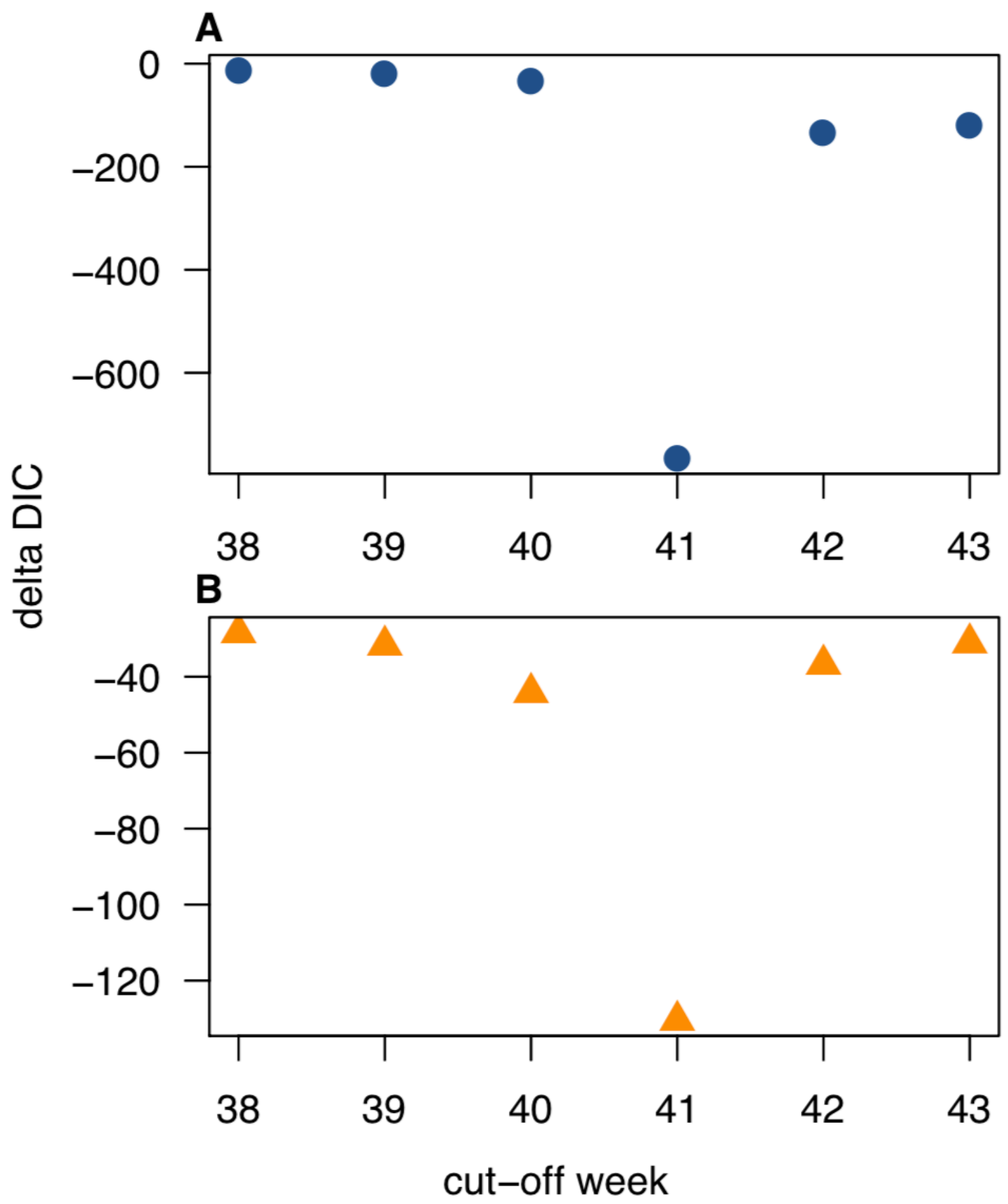

Fig S6. Model fit as a function of the timing of changed RDT performance for pneumonic (PP) (A) and bubonic plague (BP) (B). DIC is the deviance information criterium, with lower values indicating a better model fit. Delta DIC denotes the difference between the baseline model (no change in RDT performance) and the extended models (step-change in RDT performance) with different cut-off times that this change may have occurred. As elsewhere in the manuscript, the timing of samples is determined by the date of symptom onset. 

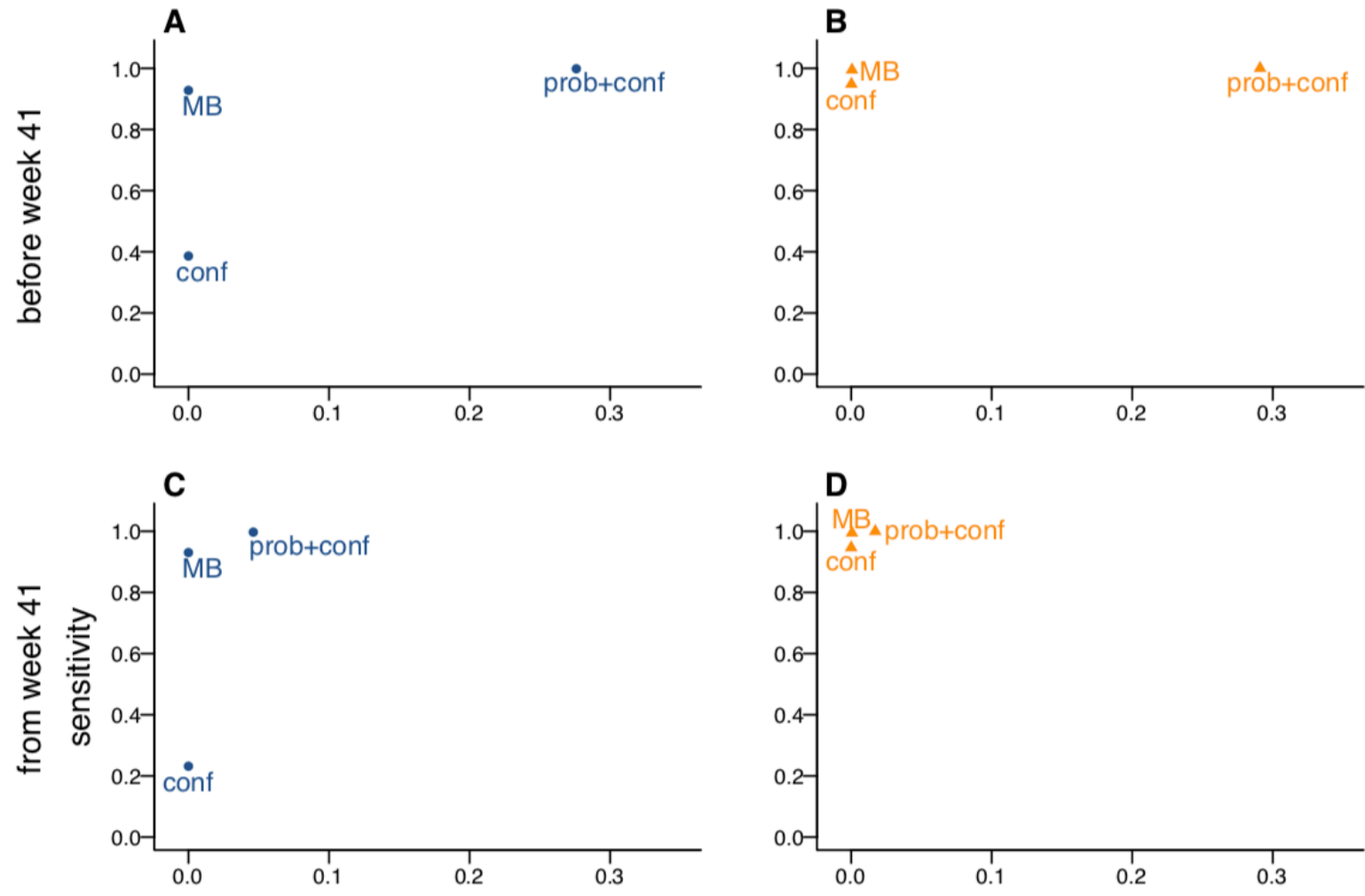

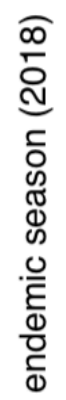

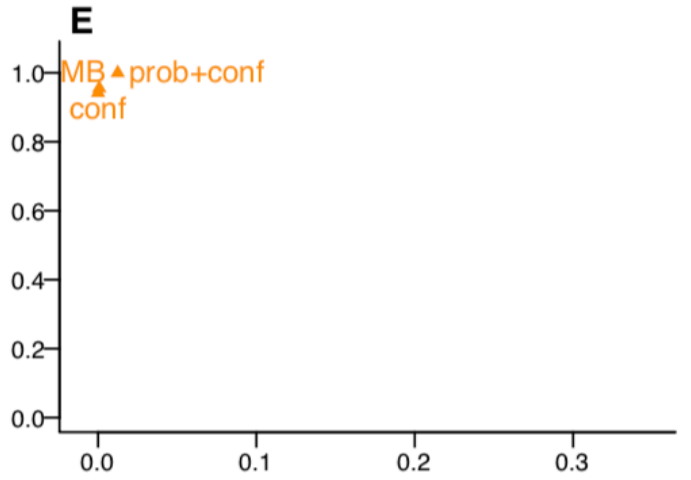




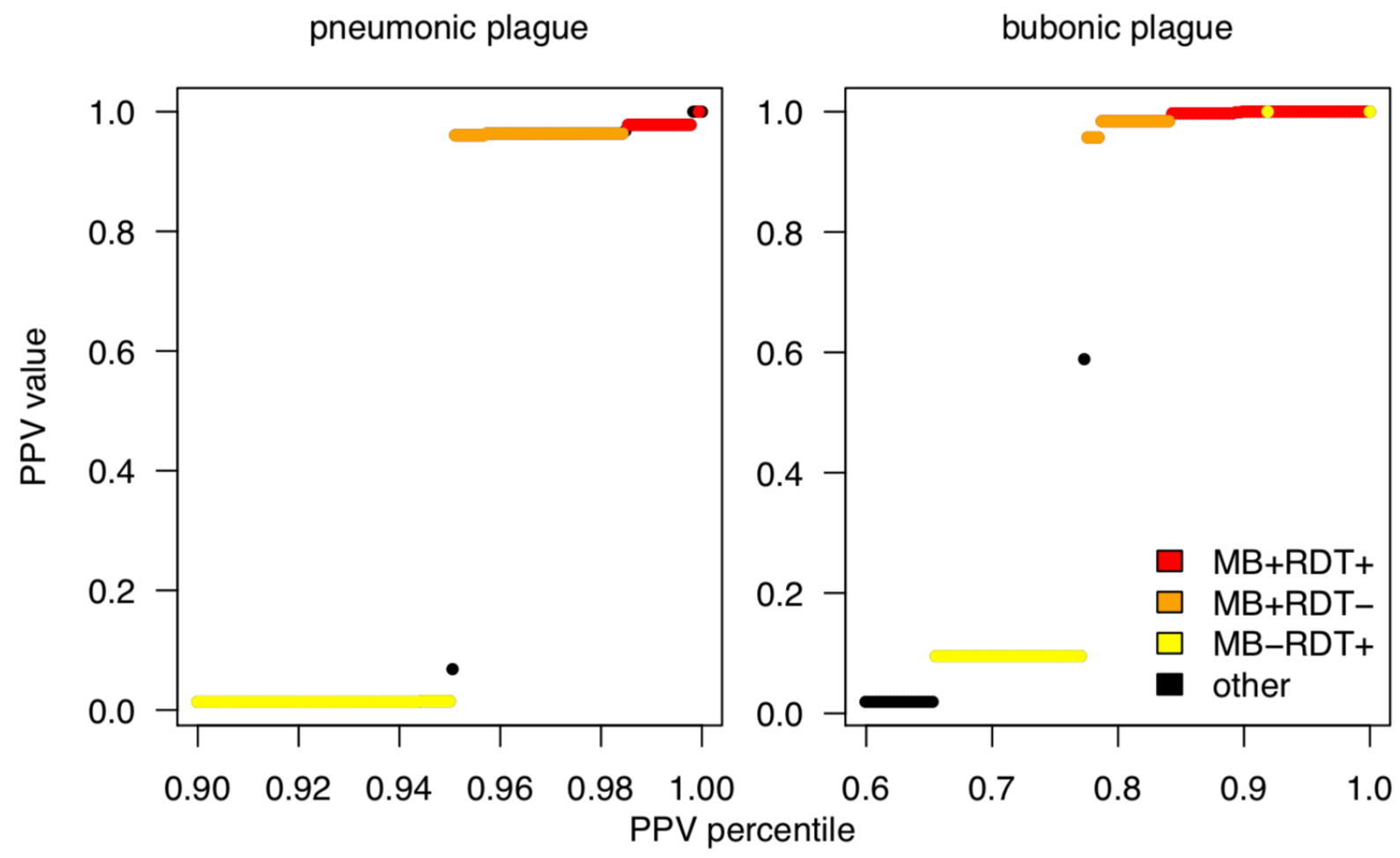

Fig S8. Distribution of positive predictive values (PPV) by test result and clinical form. The probability of being infected with Yersinia pestis given a test result is calculated based on the estimated infection prevalence among notified cases and the estimated sensitivity and specificity of the diagnostic tests. Colors denote the results for molecular biology (MB) and rapid diagnostic tests (RDT). 


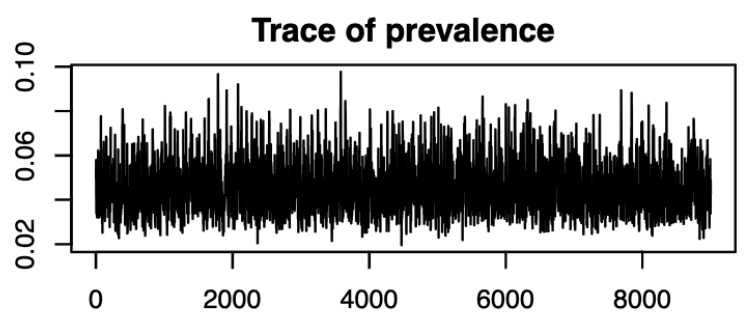

Trace of culture sensitivity

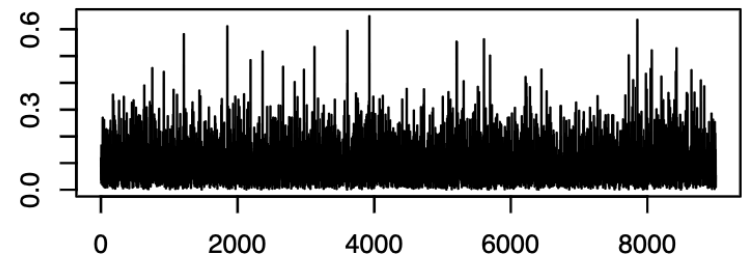

Trace of qPCR-caf sensitivity
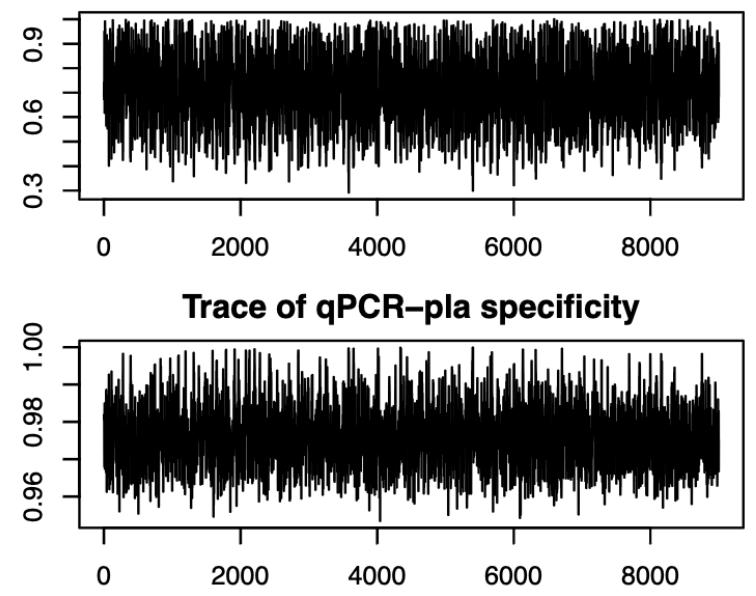

Trace of PCR-conf sensitivity

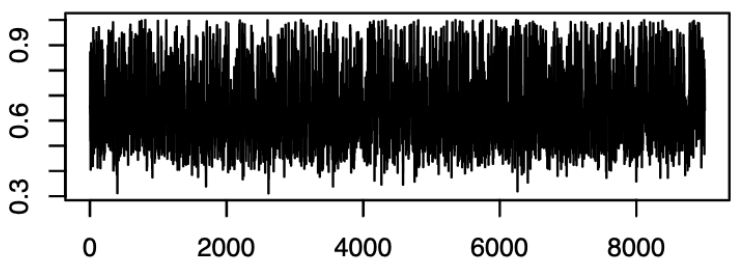

Trace of RDT sensitivity

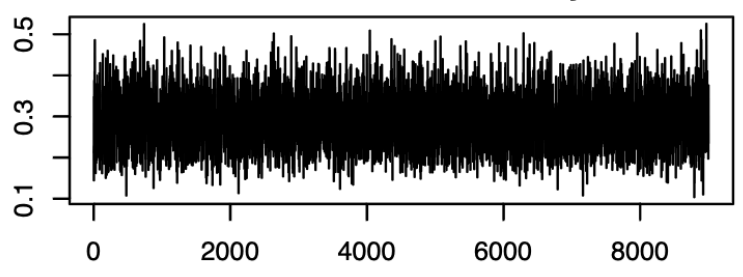

Trace of qPCR-pla sensitivity

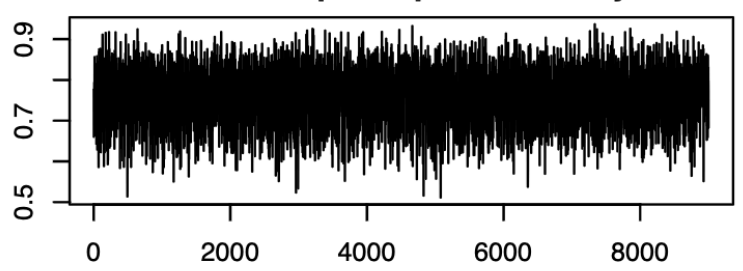

Trace of RDT specificity

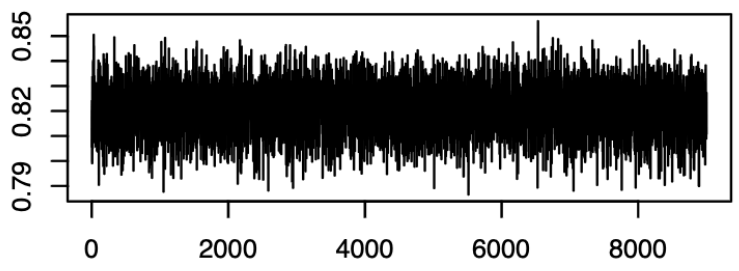

Trace of qPCR-caf specificity

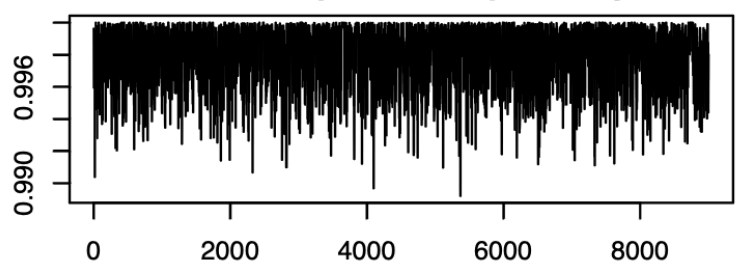

Trace of PCR-conf specificity

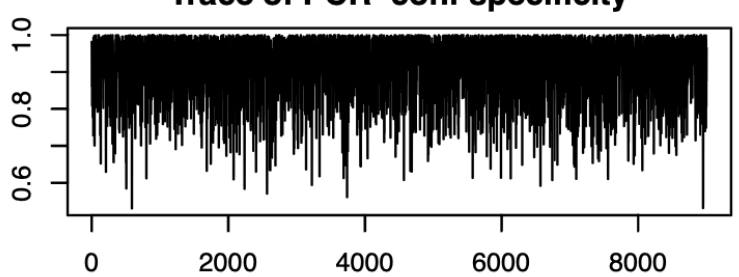


Trace of prevalence
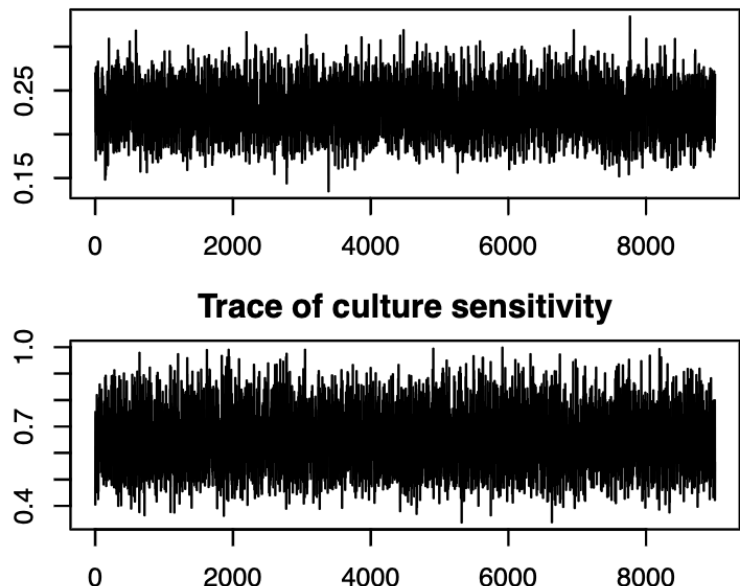

Trace of qPCR-caf sensitivity

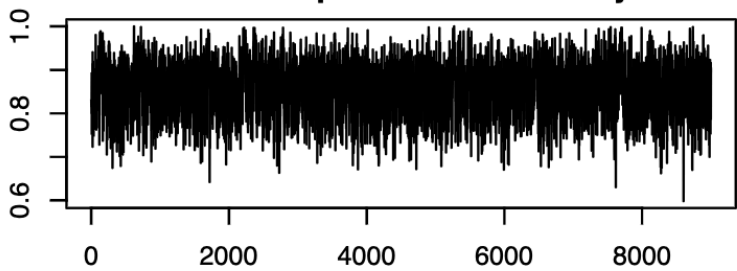

Trace of qPCR-pla specificity

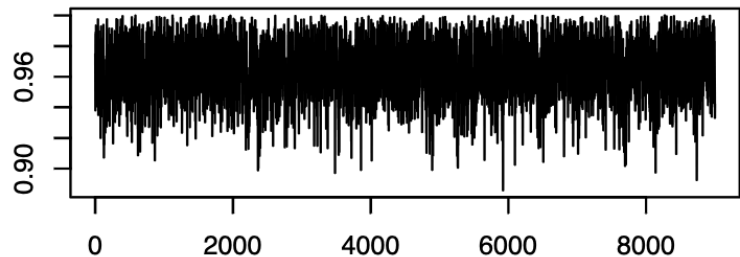

Trace of PCR-conf sensitivity

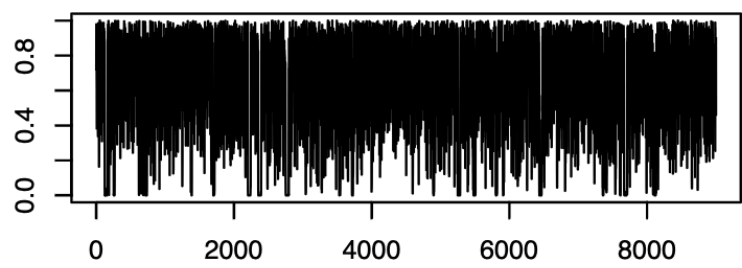

Trace of RDT sensitivity

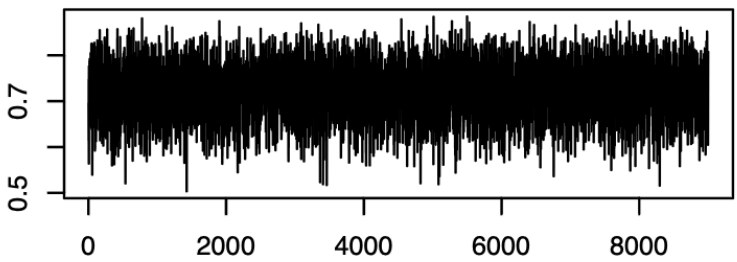

Trace of qPCR-pla sensitivity

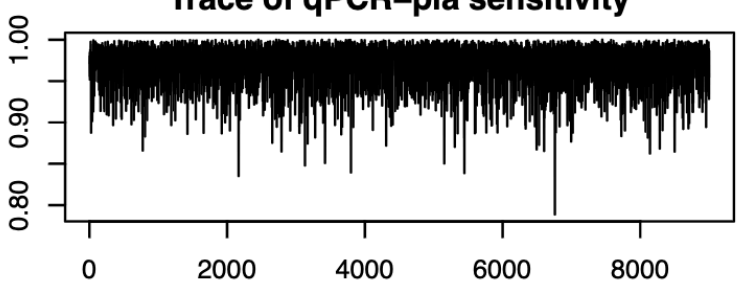

Trace of RDT specificity
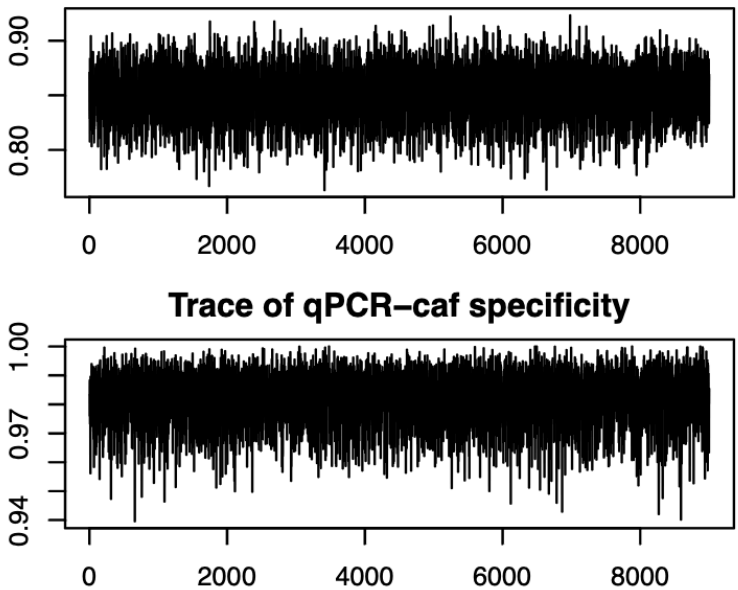

Trace of PCR-conf specificity

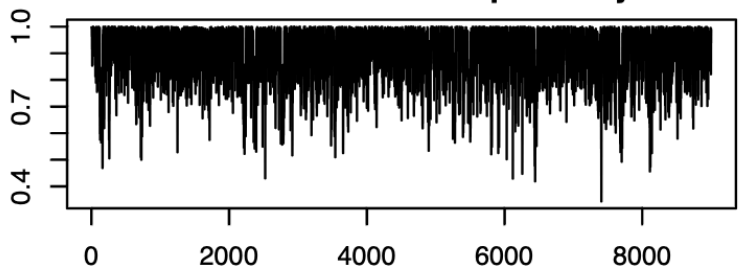


Diagnostic Sens Spec

Outcome

\begin{tabular}{|c|c|c|c|c|}
\hline & Pneumonic & Bubonic & Pneumonic & Bubonic \\
\hline$R D T$ & $28 \%(18-41)$ & $72 \%$ ( 61- 83) & $82 \%(80-84)$ & $85 \%$ ( 81- 89) \\
\hline Culture & $7 \%(0-23)$ & $64 \%(46-85)$ & Fixed at $100 \%$ & Fixed at $100 \%$ \\
\hline $\begin{array}{r}\text { Molecular } \\
\text { Biology }\end{array}$ & $80 \%(61-97)$ & $95 \%(86-100)$ & $100 \%(99-100)$ & $100 \%(98-100)$ \\
\hline$q P C R^{2}$ & $54 \%(32-76)^{3}$ & $84 \%(72-95)^{3}$ & $100 \%(100-100)^{4}$ & $100 \%(100-100)^{4}$ \\
\hline qPCR pla & $76 \%(63-88)$ & $98 \%$ ( 93-100 ) & $98 \%(96-99)$ & $97 \%$ ( $94-100$ ) \\
\hline$q P C R$ caf1 & $71 \%$ ( 48- 98 ) & $86 \%$ ( 75- 97 ) & $100 \%$ ( 99-100 ) & $98 \%(97-100)$ \\
\hline $\begin{array}{r}C P C R^{5} \\
\text { (confirmatory) }\end{array}$ & $63 \%(44-95)$ & $69 \%(0-97)$ & $95 \%(78-100)$ & $94 \%(72-100)$ \\
\hline $\begin{array}{l}{ }^{1} \text { Molecular biology alg } \\
\text { caf1, inv }{ }_{1100} \text { genes }{ }^{2} \mathrm{qP} \\
\text { result considered wher } \\
\text { aPCR result is inconclu }\end{array}$ & $\begin{array}{l}\text { thm based on } \mathrm{qP} \\
\text { based on pla anc } \\
\text { either genes test }\end{array}$ & $\begin{array}{l}\text { enes pla and caf } 1 \\
\text { enes, }{ }^{3} \text { positive res } \\
2,{ }^{5} \text { confirmatory c }\end{array}$ & $\begin{array}{l}\text { pon discordance, co } \\
\text { sidered when both } g\end{array}$ & $\begin{array}{l}\text { matory CPCR on pla, } \\
\text { s test positive, }{ }^{4} \text { negativ } \\
\text { enes. Performed when }\end{array}$ \\
\hline
\end{tabular}
caf1, inv ${ }_{1100}$ genes ${ }^{2}$ qPCR based on pla and caf1 genes, ${ }^{3}$ positive result considered when both genes test positive, ${ }^{4}$ negative qPCR result is inconclusive. 

Results of this test were removed from the final analysis because performance of that test were too low. The results of the initial CPCR were not considered in the case classification.

Diagnostic Sens Spec

Outcome

\begin{tabular}{|c|c|c|c|c|}
\hline & Pneumonic & Bubonic & Pneumonic & Bubonic \\
\hline$R D T$ & $27 \%(17-39)$ & $73 \%(63-83$ ) & $82 \%(80-84)$ & $85 \%(81-89$ ) \\
\hline $\begin{array}{r}\text { CPCR pla } \\
\text { (initial) }\end{array}$ & $86 \%$ ( 73- 97 ) & $95 \%(86-100)$ & $55 \%(52-57)$ & $62 \%(55-69)$ \\
\hline Culture & $7 \%(0-20)$ & $65 \%(47-86)$ & Fixed at $100 \%$ & Fixed at $100 \%$ \\
\hline $\begin{array}{r}\text { Molecular } \\
\text { Biology }\end{array}$ & $78 \%(52-96)$ & $95 \%(87-100)$ & $100 \%(99-100)$ & $100 \%(97-100)$ \\
\hline$q P C R^{2}$ & $48 \%(30-77)^{3}$ & $86 \%(75-97)^{3}$ & $100 \%(100-100)^{4}$ & $100 \%(100-100)^{4}$ \\
\hline qPCR pla & $81 \%$ ( 69-100 ) & $98 \%$ ( 94-100 ) & $98 \%(97-100)$ & $96 \%(92-99)$ \\
\hline qPCR caf1 & $58 \%$ ( 39- 77 ) & $88 \%(78-99)$ & $100 \%$ ( 99-100 ) & $98 \%(97-100)$ \\
\hline $\begin{array}{r}C P C R^{5} \\
\text { ifirmatory) }\end{array}$ & $70 \%(0-96)$ & $68 \%(0-97)$ & $95 \%(50-100)$ & $93 \%(68-100)$ \\
\hline
\end{tabular}

${ }^{1}$ Molecular biology algorithm based on qPCR on genes pla and caf1 with, upon discordance, confirmatory cPCR on pla, caf1, inv $v_{1100}$ genes ${ }^{2}$ qPCR based on pla and caf1 genes, ${ }^{3}$ positive result considered when both genes test positive, ${ }^{4}$ negative result considered when neither genes test positive, ${ }^{5}$ confirmatory cPCR based on pla, caf1, inv ${ }_{1100}$ genes. Performed when qPCR result is inconclusive. 

outcomes, in a scenario with a change in RDT performance at week 41 of the outbreak.

Diagnostic Sens Spec

Outcome

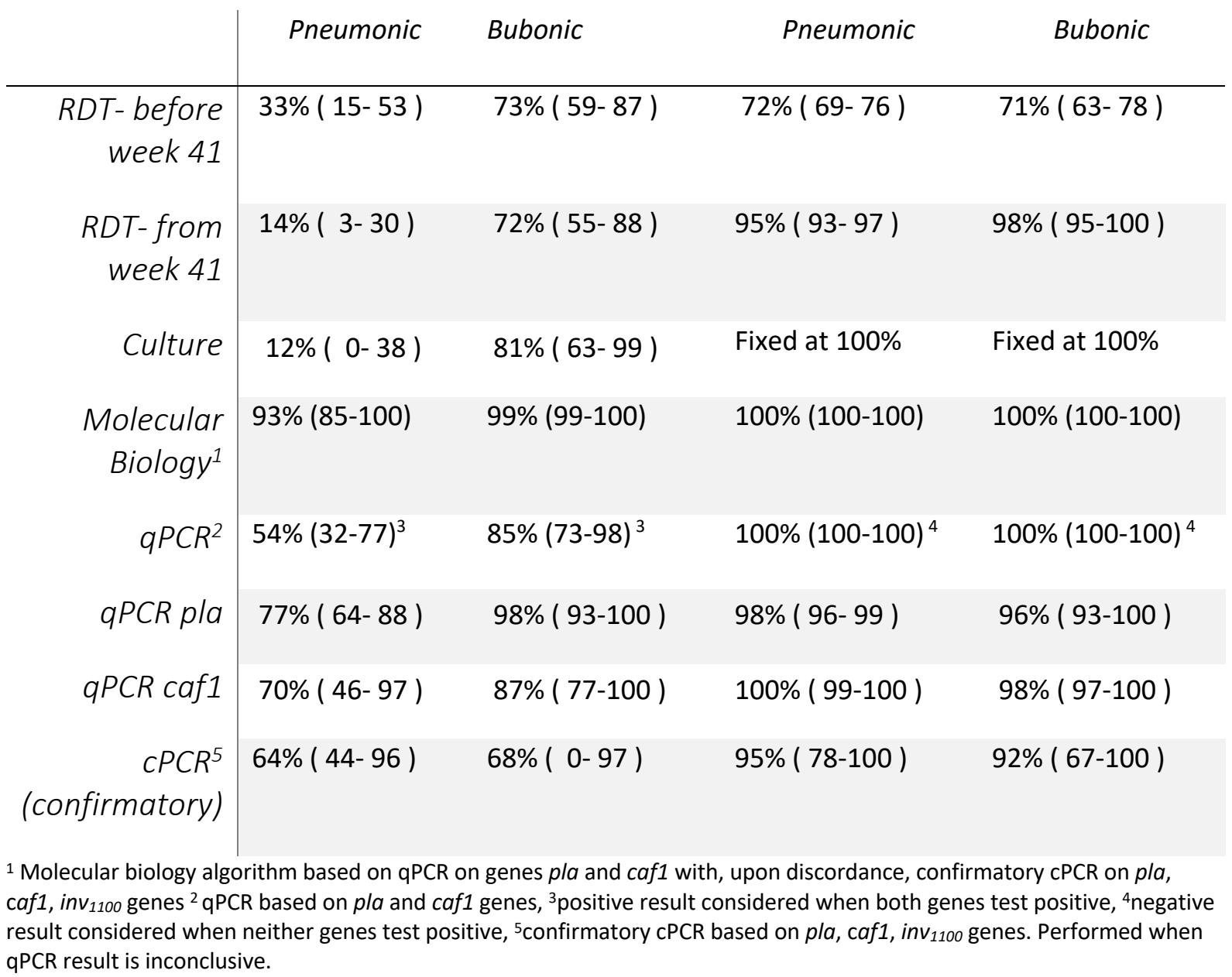

${ }_{1}^{1}$ Molecular biology algorithm based on qPCR on genes pla and caf1 with, upon discordance, confirmatory cPCR on pla, 
Table S4. Model estimates of the performance of RDT, culture, MB, and of tests that would be based on single diagnostic outcomes, with a non-informative uniform prior from 0 to 1 on the prevalence of infection among notified cases.

Diagnostic Sens Spec

Outcome

\begin{tabular}{|c|c|c|c|c|}
\hline & Pneumonic & Bubonic & Pneumonic & Bubonic \\
\hline$R D T$ & $28 \%(18-40)$ & $73 \%(62-83$ ) & $82 \%(80-84)$ & $86 \%(81-90)$ \\
\hline Culture & $7 \%(0-23)$ & $66 \%(47-87)$ & Fixed at $100 \%$ & Fixed at $100 \%$ \\
\hline $\begin{array}{r}\text { Molecular } \\
\text { Biology }\end{array}$ & $81 \%(59-100)$ & $90 \%(72-100)$ & $100 \%(99-100)$ & $100 \%$ (99-100) \\
\hline$q P C R^{2}$ & $53 \%(32-75)^{3}$ & $84 \%(72-94)^{3}$ & $100 \%(100-100)^{4}$ & $100 \%(100-100)^{4}$ \\
\hline qPCR pla & $76 \%(64-88$ ) & $98 \%(93-100)$ & $98 \%(96-99)$ & $97 \%$ ( 94-100 ) \\
\hline qPCR caf1 & $70 \%$ ( 46- 95 ) & $86 \%(81-90$ ) & $100 \%$ ( 99-100 ) & $98 \%(97-100)$ \\
\hline $\begin{array}{r}c P C R^{5} \\
\text { (confirmatory) }\end{array}$ & $63 \%(44-96)$ & $71 \%(15-100)$ & $95 \%(79-100$ ) & $94 \%(73-100)$ \\
\hline
\end{tabular}

